\title{
Gibbs States of a One Dimensional Quantum Lattice
}

\author{
HUZIHIRo ARAKI* \\ I.H.E.S., 91 - Bures-sur-Yvette, France
}

Received June 20, 1969

\begin{abstract}
A one dimensional infinite quantum spin lattice with a finite range interaction is studied. The Gibbs state in the infinite volume limit is shown to exist as a primary state of a UHF algebra. The expectation value of any local observables in the state as well as the mean free energy depend analytically on the potential, showing no phase transition. The Gibbs state is an extremal KMS state.
\end{abstract}

\section{§ 1. Introduction}

A one dimensional infinite classical spin lattice system has been studied in [12] and shown to be without any phase transition for a large class of interactions. We show an analogous result for the quantum case with any finite range interaction.

We first show that the power series for the time displacement automorphism of the algebra of observables has an infinite radius of convergence for local observables in one dimensional lattice. This enables us to use the Tomonaga-Schwinger-Dyson perturbation type formula and pull out each potential from $e^{-\beta H}$ as a factor. The transfer matrix technique for the classical one dimensional Ising model is then applicable in a fashion analogous to [12] and we obtain a formula for the infinite volume Gibbs state in terms of an eigen state of a certain linear bounded operator acting on observables.

A standard pertubation theory of bounded linear operators on a Banach space enables us to find an analytic continuation of the Gibbs state with respect to the interaction potential and to prove the analyticity of the expectation value of local observables in the Gibbs state as well as the analyticity of the mean free energy.

This technique is applicable also to the classical case, provided that the interaction potential decreases exponentially at large separation.

The Gibbs state is shown to be invariant under time and lattice translation, satisfies the KMS boundary condition and has the exponen-

* On leave from Research Institute for Mathematical Sciences, Kyoto University Kyoto, Japan. 
tial, uniform clustering property. The last property implies, by a general theorem, that the state is primary (a factor state) and is an extremal KMS state.

\section{§ 2. Notation and Results}

We represent a one dimensional lattice by the set of integers $Z=\{0, \pm 1, \ldots\}$. For each lattice point $j$, we have a $d$-dimensional Hilbert space $\mathscr{H}_{j}$ where $d$ is finite and independent of $j$. For each finite subset $I$ of $Z$, we consider a finite dimensional full matrix algebra $\mathfrak{U}(I)=\mathscr{B}(\mathscr{H}(I)), \mathscr{H}(I)=\bigotimes_{j \in I} \mathscr{H}_{j}$. For $I \subset I^{\prime}, Q \in \mathfrak{A}(I)$ is identified with $Q \otimes 1_{I^{\prime} \backslash I}$ in $\mathfrak{U}\left(I^{\prime}\right)$ where $I^{\prime} \backslash I$ denotes the complement of $I$ in $I^{\prime}$ and $1_{I^{\prime} \backslash I}$ is the identity in $\mathfrak{A}\left(I^{\prime} \backslash I\right)$. The collection of $\mathfrak{A}(I)$ for all finite subsets $I$ of $Z$ together with this identification defines a normed *-algebra $\mathfrak{A}_{0}$ (the algebra of local observables). Its completion $\mathfrak{A}$ is taken as the $C^{*}$-algebra of quasi local observables. The closed *-subalgebra of $\mathfrak{A}$ generated by all $\mathfrak{A}(I), I \subset I^{\prime}$ will be denoted by $\mathfrak{A}\left(I^{\prime}\right)$ for an infinite subset $I^{\prime}$ of $Z$ (as well as for a finite subset $I^{\prime}$ ).

For any two lattice points $j$ and $j^{\prime}$, we fix a unitary mapping $w\left(j, j^{\prime}\right)$ from $\mathscr{H}_{j^{\prime}}$ onto $\mathscr{H}_{j}$ such that $w\left(j, j^{\prime}\right) w\left(j^{\prime}, j^{\prime \prime}\right)=w\left(j, j^{\prime \prime}\right), w\left(j, j^{\prime}\right)^{*}=w\left(j^{\prime}, j\right)$ and $w(j, j)=1$. Let $I+a$ denote the set $\{j+a ; j \in I\}$. Let $w(I+a, I)=\bigotimes_{j \in I} w(j+a, j)$, which is a unitary map of $\mathscr{H}(I)$ onto $\mathscr{H}(I+a)$. The *automorphism of $\mathfrak{A}$, which is induced by a ${ }^{*}$-isomorphism $Q \in \mathfrak{U}(I) \rightarrow w(I+a, I)$ $Q w(I+a, I)^{*}$, is denoted by $\tau_{s}(a)$ and is called a lattice translation. We also need the *-isomorphism of $\mathfrak{A}(Z \backslash[1-n, n])$ onto $\mathfrak{A}$, which is induced by the *-isomorphisms $\tau_{s}(n) \otimes \tau_{s}(-n)$ of $\mathfrak{A}([n+1, N])$ $\otimes \mathfrak{U}([1-N,-n]) \quad$ onto $\quad \mathfrak{U}([1, N-n]) \otimes \mathfrak{U}([1-N+n, 0]), \quad N=n+1$, $n+2, \ldots$ It is denoted by $\tau_{c}(n)$. Here $[a, b]$ denotes the set of integers $j$ satisfying $a \leqq j \leqq b$.

Let $\Phi(I)$ be the interaction potential among lattice sites in $I . \Phi(I)^{*}$ $=\Phi(I), \Phi(I) \in \mathfrak{A}(I)$. We require $\Phi(I)=0$ if $I$ is not within some interval of length $r$. We also require $\Phi(I+a)=\tau_{s}(a) \Phi(I)$. The Hamiltonian for a finite interval $\left[N_{1}, N_{2}\right]$ is

$$
U\left(N_{1}, N_{2}\right)=\sum_{I \subset\left[N_{1}, N_{2}\right]} \Phi(I) .
$$

The Gibbs state $\varphi_{a, b}^{G}$ of $\mathfrak{A}([a, b])$ is

$$
\begin{aligned}
\varphi_{a, b}^{G}(Q) & =Z(a, b)^{-1} \operatorname{tr}_{a b}[Q \exp -U(a, b)], \\
Z(a, b) & =\operatorname{tr}_{a b}[\exp -U(a, b)],
\end{aligned}
$$

where $\operatorname{tr}_{a b}$ is the trace of a full matrix algebra $\mathfrak{A}([a, b])$. 
Theorem 2.1. (i) The following limit exists and is finite:

$$
P(\Phi)=\lim _{b \rightarrow a \rightarrow \infty}(b-a)^{-1} \log Z(a, b) .
$$

It is holomorphic in $\Phi$.

(ii) For every $Q \in A_{0}$, the following limit exists and defines a state of $\mathfrak{A}$ :

$$
\varphi_{\Phi}(Q)=\lim _{a \rightarrow-\infty, b \rightarrow+\infty} \varphi_{a b}^{G}(Q) .
$$

For each $Q \in \mathfrak{A}_{0}$, it is holomorphic in $\Phi$.

The holomorphy in $\Phi$ means the real holomorphy in $\zeta=\left(\zeta_{1}, \ldots, \zeta_{n}\right)$ when each $\Phi(I)=\Phi(I, \xi)$ is a restriction of an $\mathfrak{A}(I)$ valued holomorphic function $\Phi(I, \zeta)$ to $\zeta=\xi \in$ some real domain.

The analyticity statement can be proved for the following class of quasilocal observables with exponential tail.

Definition 2.2. Let $Q \in \mathfrak{U}$.

$$
\begin{aligned}
\|Q\|_{n} & =\inf _{Q_{n} \in \mathfrak{H}([-n, n])}\left\|Q-Q_{n}\right\| . \\
\|Q\|_{n, x} & =\sum_{k=n}^{\infty} x^{k}\|Q\|_{k} . \\
\|Q\|_{n, x} & =\|Q\|+\|Q\|_{n, x} .
\end{aligned}
$$

For $x>1, \mathfrak{U}(x)$ is the set of $Q \in \mathfrak{A}$ such that $\|Q\|_{n, x}<\infty$.

$\varphi_{\Phi}(Q)$ is holomorphic in $\Phi$ if $Q \in \mathfrak{A}(x)$ for some $x>1$.

The limit

$$
\tau_{T}(t) Q=\lim _{a \rightarrow-\infty, b \rightarrow \infty} e^{i t U(a, b)} Q e^{-i t U(a, b)}
$$

exists in $\mathfrak{A}$ for all $Q \in \mathfrak{A}$ and defines a continuous one parameter group of * automorphisms of $\mathfrak{A}$, which we denote by $\tau_{T}(t)$ and call a time translation. (The unit of time is $(\beta \hbar)^{-1}$.) A state $\varphi$ of $\mathfrak{A}$ is time and lattice translation invariant if $\varphi\left(\tau_{T}(t) \tau_{s}(a) Q\right)=\varphi(Q)$, for any $t, a$ and $Q \in \mathfrak{A}$. It satisfies the KMS boundary condition if $\varphi\left(Q_{1} Q_{2}\left(f_{0}\right)\right)=\varphi\left(Q_{2}\left(f_{1}\right) Q_{1}\right)$ for all $Q_{1}, Q_{2} \in \mathfrak{A}$ and $f \in \mathscr{D}$ where $Q_{2}\left(f_{\alpha}\right)=\int \tau_{T}(t) Q_{2} f_{\alpha}(t) \mathrm{dt}, f_{\alpha}(t)$ $=\int_{-\infty}^{\infty} e^{-i s t+\alpha s} f(s)$ ds. Let $S_{1}$ be a convex subset of set of states of $\mathfrak{A}$. A state $\varphi \in S_{1}$ is extremal in $S_{1}$ if $\varphi=\lambda \varphi_{1}+(1-\lambda) \varphi_{2}, \varphi_{1}, \varphi_{2} \in S_{1}, 0<\lambda<1$ imply $\varphi_{1}=\varphi_{2}=\varphi$. If $S_{1}$ is the set of translation invariant states, $\varphi$ is an extremal translation invariant state and if $S_{1}$ is the set of time translation invariant states satisfying the KMS boundary condition, then $\varphi$ is an extremal KMS state. 
A state $\varphi$ has an exponential clustering property if there exists $\varrho>0$ such that

$$
\lim _{n \rightarrow \infty} e^{\varrho|n|}\left[\varphi\left(Q_{1} \tau_{s}(n) Q_{2}\right)-\varphi\left(Q_{1}\right) \varphi\left(\tau_{s}(n) Q_{2}\right)\right]=0
$$

for any fixed $Q_{1}, Q_{2} \in \mathfrak{A}_{0} . \varphi$ has a uniform clustering property if there exists $N$ for any given $\varepsilon>0$ and $Q_{1} \in \mathfrak{A}$ such that

$$
\left|\varphi\left(Q_{1} Q_{2}\right)-\varphi\left(Q_{1}\right) \varphi\left(Q_{2}\right)\right|<\varepsilon\left\|Q_{2}\right\|
$$

for all $Q_{2} \in \mathfrak{U}(Z \backslash[-N, N])$. A state $\varphi$ is primary (or a factor state) if the cyclic representation $\pi_{\varphi}$ of $\mathfrak{A}$ associated with $\varphi$ through the GNS construction is such that $\pi_{\varphi}(\mathfrak{Q})^{\prime \prime}$ is a factor (i.e. the center of $\pi_{\varphi}(\mathfrak{U})^{\prime \prime}$ consists of multiples of the identity).

Theorem 2.3. The Gibbs state $\varphi_{\Phi}$ for the infinite system is invariant under time and lattice translation, satisfies the KMS boundary condition, has exponential and uniform clustering properties, is primary, is an extremal KMS state, and is an extremal lattice translation invariant state.

In the following discussion, we use the following combination of $\Phi(I)$ :

$$
\Phi=\sum_{I \subset[0, r]} n_{r}(I)^{-1} \Phi(I)
$$

where $n_{r}(I)$ is the number of the translates $I+a$ of $I$ which is still in $[0, r]$. We denote

$$
\begin{aligned}
H(I) & =\sum_{[n, r+n] \subset I} \tau_{s}(n) \Phi, \\
H(a, b) & =H([a, b]) .
\end{aligned}
$$

$H(a, b)$ and $U(a, b)$ differs only near the two ends:

$$
\begin{gathered}
U(a, b)-H(a, b)=\Delta_{a}^{-}+\Delta_{b}^{+}, \\
\Delta_{b}^{+} \in A([b-r, b]), \quad \Delta_{a}^{-} \in A([a, a+r]), \\
\left\|\Delta_{b}^{+}\right\| \leqq \sum_{I \subset[0, r]}\|\Phi(I)\|, \\
\left\|\Delta_{a}^{-}\right\| \leqq \sum_{I \subset[0, r]}\|\Phi(I)\| .
\end{gathered}
$$

\section{§ 3. The Spaces $\mathfrak{A}(M, x)$}

Lemma 3.1. $\|Q\|_{n, x}$ is a norm of linear space $\mathfrak{A}(x)$. $\mathfrak{A}(x)$ equipped with the norm || $\mid \|_{n, x}$ (denoted as $\left.\mathfrak{U}(n, x)\right)$ is a* Banach algebra.

Proof. There always exists $Q^{(n)} \in \mathfrak{A}([-n, n])$ such that $\left\|Q-Q^{(n)}\right\|=\|Q\|_{n}$ due to the compactness of a bounded closed subset of $A([-n, n])$. Let $9^{*}$ 


$$
\begin{aligned}
& \left\|Q_{1}-Q_{1}^{(n)}\right\|=\left\|Q_{1}\right\|_{n},\left\|Q_{2}-Q_{2}^{(n)}\right\|=\left\|Q_{2}\right\|_{n} \text {. Then } \\
& \left\|Q_{1}+Q_{2}\right\|_{n} \leqq\left\|Q_{1}+Q_{2}-Q_{1}^{(n)}-Q_{2}^{(n)}\right\| \leqq\left\|Q_{1}\right\|_{n}+\left\|Q_{2}\right\|_{n} .
\end{aligned}
$$

Similarly, $\|C Q\|_{n} \leqq|C|\|Q\|_{n}$. At the same time, $\|Q\|_{n} \leqq\left|C^{-1}\right|\|C Q\|_{n}$. Therefore $\|C Q\|_{n}=|C|\|Q\|_{n}$.

Obviously $\left\|Q^{*}\right\|_{n, x}=\|Q\|_{n, x}$. Further, $\left\|Q_{1} Q_{2}\right\|_{n} \leqq\left\|Q_{1} Q_{2}-Q_{1}^{(n)} Q_{2}^{(n)}\right\|$ $\leqq\left\|Q_{1}\right\|\left\|Q_{2}\right\|_{n}+\left\|Q_{2}^{(n)}\right\|\left\|Q_{1}\right\|_{n} \leqq\left\|Q_{1}\right\|_{n}\left\|Q_{2}\right\|_{n}+\left\|Q_{1}\right\|\left\|Q_{2}\right\|_{n}+\left\|Q_{1}\right\|_{n}\left\|Q_{2}\right\|$. Hence $\left\|Q_{1} Q_{2}\right\|_{n, x} \leqq\left\|Q_{1}\right\|_{n, x}\left\|Q_{2}\right\|_{n, x}$.

Any Cauchy sequence $Q_{k}$ with respect to \|\|$_{n, x}$ is a Cauchy sequence with respect to the norm in $\mathfrak{A}$ and has a limit $Q$ in $\mathfrak{A}: \lim _{k}\left\|Q_{k}-Q\right\|=0$. This implies $\|Q\|_{n} \leqq \varliminf_{k}\left\|Q_{k}\right\|_{n} \leqq \varlimsup_{k}\left\|Q_{k}\right\|_{n} \leqq\|Q\|_{n}$ and hence $\|Q\|_{n}$ $=\lim _{k}\left\|Q_{k}\right\|_{n}$. Hence

$$
\|Q\|+\sum_{l=n}^{N} x^{l}\|Q\|_{l}=\lim _{k \rightarrow \infty}\left(\left\|Q_{k}\right\|+\sum_{l=n}^{N} x^{l}\left\|Q_{k}\right\|_{l}\right) \leqq \sup _{k}\left\|Q_{k}\right\|_{n, x}<\infty .
$$

Thus $\|Q\|_{n, x}<\infty$ and $Q \in \mathfrak{A}(n, x)$. Given $\varepsilon>0$. There exists $K$ such that $\left\|Q_{K}-Q_{k}\right\|_{n, x}<\varepsilon / 4$ for $k \geqq K$. There exists $N$ such that $\left\|Q_{K}\right\|_{N, x}<\varepsilon / 4$ and $\|Q\|_{N, x}<\varepsilon / 4$. There exists $K^{\prime}$ such that $\left\|Q-Q_{k}\right\|+\sum_{l=n}^{N-1} x^{l}\left\|Q-Q_{k}\right\|_{l}$ $<\varepsilon / 4$ for $k \geqq K^{\prime}$. Then $\left\|Q-Q_{k}\right\|_{n, x}<\varepsilon$ for $k \geqq \max \left(K, K^{\prime}\right)$. Therefore $\lim _{k}\left\|Q_{k}-Q\right\|_{n, x}=0$. Q.E.D.

Lemma 3.2. The set $\Sigma_{N}(\gamma)$ of $Q \in \mathfrak{A}$ such that

$$
\|Q\| \leqq \gamma_{0}, \quad\|Q\|_{l} \leqq \gamma_{l}, \quad l=N, N+1, \ldots
$$

is convex and compact provided that $\gamma_{0}<\infty, \lim _{l \rightarrow \infty} \gamma_{l}=0$.

Proof. The convexity is straight forward from the triangular inequality. By setting $Q_{n}=0$ in (2.6), we obtain $\|Q\|_{n} \leqq\|Q\| \leqq \gamma_{0}$. Let $Q^{(n)} \in \mathfrak{A}([-n, n])$ be such that $\left\|Q-Q^{(n)}\right\|=\|Q\|_{n}$. Then $\left\|Q^{(n)}\right\| \leqq\|Q\|+\|Q\|_{n} \leqq 2 \gamma_{0}$.

Let $Q_{k} \in \Sigma_{N}(\gamma)$. Let $k(i, j), j=1,2, \ldots$ be a subsequence of $k(i-1, j)$, $j=1,2, \ldots$ such that $Q_{k(i, j)}^{(i)}$ is convergent in norm as $j \rightarrow \infty$, where $n(N-1, k)=k$ and $i=N, N+1, \ldots$. Such choice is inductively possible because $\left\|Q_{k}^{(i)}\right\| \leqq 2 \gamma_{0}$ for all $i$ and $k$.

We can show that $Q_{k(j, j)}$ is a Cauchy sequence in $\mathfrak{A}$. Let $\varepsilon>0$ be given. There exist $K$ such that $\gamma_{l}<\varepsilon / 3$ for $l \geqq K$ and $K^{\prime}>K$ such that $\left\|Q_{k(K, j)}^{(K)}-Q_{k\left(K, j^{\prime}\right)}^{(K)}\right\|<\varepsilon / 3$ for $j, j^{\prime} \geqq K^{\prime}$. Since $k(j, j)=k(K, v)$ with $v \geqq j$ if $j \geqq K$, we have $\left\|Q_{k(j, j)}^{(K)}-Q_{k\left(j^{\prime}, j^{\prime}\right)}^{(K)}\right\|<\varepsilon / 3$ if $j, j^{\prime} \geqq K^{\prime}$. Hence

$$
\begin{gathered}
\left\|Q_{k(j, j)}-Q_{k\left(j^{\prime}, j^{\prime}\right)}\right\| \leqq\left\|Q_{k(j, j)}-Q_{k(j, j)}^{(K)}\right\| \\
+\left\|Q_{k(j, j)}^{(K)}-Q_{k\left(j^{\prime}, j^{\prime}\right)}^{(K)}\right\|+\left\|Q_{k\left(j^{\prime}, j^{\prime}\right)}^{(K)}-Q_{k\left(j^{\prime}, j^{\prime}\right)}\right\|<\varepsilon
\end{gathered}
$$

if $j, j^{\prime} \geqq K^{\prime}$. Hence $Q_{k}$ has a convergent subsequence $Q_{k(j, j)}$. 
Since $\mathfrak{A}$ is a separable Banach space, its sequentially compact subset is compact. Q.E.D.

Lemma 3.3. Let $x_{2}>x_{1}>1$. The closure of a bounded subset of $\mathfrak{U}\left(n, x_{2}\right)$ with respect to the norm in $\mathfrak{U}$ is in $\mathfrak{A}\left(n, x_{2}\right)$ and is a compact subset of $\mathfrak{A}\left(n, x_{1}\right)$ (with respect to \|\|$\left.\|_{n, x_{1}}\right)$.

Proof. Let $\left\|Q_{k}\right\|_{n, x_{2}} \leqq a$. Then $\left\|Q_{k}\right\| \leqq a,\left\|Q_{k}\right\|_{l} \leqq x_{2}^{-l} a$ for $l=n, n+1, \ldots$ and $\lim _{l \rightarrow \infty} x_{2}^{-l} a=0$. Hence $Q_{k}$ has a subsequence which converges with respect to the norm in $\mathfrak{A}$. Let now $Q_{k}$ be a sequence such that $\lim _{k \rightarrow \infty}\left\|Q-Q_{k}\right\|$ $=0$. By the latter half of the proof of Lemma 3.1, $\|Q\|_{n}=\lim _{k}\left\|Q_{k}\right\|_{n}$ and hence $\|Q\|+\sum_{l=n}^{N-1}\|Q\|_{l} \leqq a$. Therefore $\|Q\|_{n, x_{2}} \leqq a$. Since $\left\|Q_{k}\right\|_{l} \leqq x_{2}^{-l} a$, $l \geqq n$, we have $\left\|Q_{k}\right\|_{N, x_{1}} \leqq a\left(x_{1} / x_{2}\right)^{N}\left(1-\left(x_{1} / x_{2}\right)\right)^{-1}$, which tend to 0 as $N \rightarrow \infty$. This is true also when $Q_{k}$ is replaced by $Q$. Since $\lim \left\|Q-Q_{k}\right\|_{l}=0$ for each $l$, we have $\lim \left\|Q-Q_{k}\right\|_{n, x_{1}}=0$. Q.E.D.

In the above discussion $\mathfrak{U}(n, x)$ for given $x$ and varying $n$ are topologically equivalent. We introduced $\|\mid\|_{n, x}$ merely for the convenience in later computation.

Definition 3.4. $\mathfrak{A}_{1}$ and $\mathfrak{A}_{2}$ are sets of $Q \in \mathfrak{A}$ satisfying the following conditions (i) and (ii), respectively.

(i) $\sum_{l} x^{l}\|Q\|_{l}<\infty$ for all $x$.

(ii) $\sup _{n} n^{-1}\left[\log \|Q\|_{n}+(n / r) \log n\right]<\infty$.

Lemma 3.5. $\mathfrak{A}_{1}$ and $\mathfrak{A}_{2}$ are ${ }^{*}$-subalgebra of $\mathfrak{A}$ stable under $\tau_{s}(a) . \mathfrak{A}_{1}$ contains $\mathfrak{A}_{2}$.

Proof. (i) Let $Q_{1}, Q_{2} \in \mathfrak{A}$. Then

$$
\begin{aligned}
\Sigma x^{l}\left\|c_{1} Q_{1}+c_{2} Q_{2}\right\|_{l} \leqq\left|c_{1}\right| \Sigma x^{l}\left\|Q_{1}\right\|_{l}+\left|c_{2}\right| \Sigma x^{l}\left\|Q_{2}\right\|_{l}, & \sup _{n} n^{-1}\left[\log \left\|c_{1} Q_{1}+c_{2} Q_{2}\right\|_{n}+(n / r) \log n\right] \\
\leqq & \sup _{n} n^{-1}\left[\log \left(\left|c_{1}\right|\left\|Q_{1}\right\|_{n}+\left|c_{2}\right|\left\|Q_{2}\right\|_{n}\right)+(n / r) \log n\right] \\
= & \max _{j=1,2}\left\{\sup _{n} n^{-1}\left[\log \left\|Q_{j}\right\|_{n}+(n / r) \log n\right]\right\}+\log \left(\left|c_{1}\right|+\left|c_{2}\right|\right) .
\end{aligned}
$$

Hence $\mathfrak{A}_{1}$ and $\mathfrak{A}_{2}$ are linear subset of $\mathfrak{\mathfrak { A }}$. Next

$$
\begin{aligned}
\left\|Q_{1} Q_{2}\right\|_{n} \leqq\left\|Q_{1} Q_{2}-Q_{1}^{(n)} Q_{2}^{(n)}\right\| \leqq & \left\|Q_{1}\right\|\left\|Q_{2}\right\|_{n}+\left\|Q_{2}^{(n)}\right\|\left\|Q_{1}\right\|_{n} \\
& \leqq\left\|Q_{1}\right\|\left\|Q_{2}\right\|_{n}+2\left\|Q_{2}\right\|\left\|Q_{1}\right\|_{n} .
\end{aligned}
$$


Hence

$$
\begin{aligned}
& \sum x^{l}\left\|Q_{1} Q_{2}\right\|_{l} \leqq\left\|Q_{1}\right\| \Sigma x^{l}\left\|Q_{2}\right\|_{l}+2\left\|Q_{2}\right\| \Sigma x^{l}\left\|Q_{1}\right\|_{l}, \\
& \sup _{n} n^{-1}\left[\log \left\|Q_{1} Q_{2}\right\|_{n}+(n / r) \log n\right] \\
& \quad \leqq \max _{j=1,2}\left\{\sup _{n} n^{-1}\left[\log \left\|Q_{j}\right\|_{n}+(n / r) \log n\right]\right\}+\log \left(\left\|Q_{1}\right\|+2\left\|Q_{2}\right\|\right) .
\end{aligned}
$$

Therefore $\mathfrak{A}_{1}$ and $\mathfrak{A}_{2}$ are algebras. Since $\left\|Q^{*}\right\|_{l}=\|Q\|_{l}, \mathfrak{A}_{1}$ and $\mathfrak{A}_{2}$ are *-algebras. $\left\|\tau_{s}(a) Q\right\|_{l} \leqq\|Q\|_{l+a}$. Therefore $\mathfrak{Q}_{1}$ and $\mathfrak{H}_{2}$ are stable under $\tau_{s}(a) . \mathfrak{U}_{2} \subset \mathfrak{U}_{1}$ is obvious. Q.E.D.

Definition 3.6. Let $Q \in \mathfrak{A}$. Define $\left\|Q^{-1}\right\|$ to be $\infty$ if $Q^{-1} \notin \mathfrak{A}$.

$$
\begin{aligned}
\alpha(Q) & =\|Q\|\left\|Q^{-1}\right\|, \\
\alpha_{n}(Q) & =\inf _{Q_{n} \in \mathscr{2}([-n, n])}\left\|Q-Q_{n}\right\|\left\|Q_{n}^{-1}\right\| .
\end{aligned}
$$

Lemma 3.7. Let $Q>0$. Then

$$
\begin{aligned}
& \left\|Q^{-1}\right\|^{-1} \leqq Q \leqq\|Q\|, \\
& \alpha(Q)=\sup \varphi^{\prime}(Q) / \varphi^{\prime \prime}(Q)
\end{aligned}
$$

where sup is taken over all states $\varphi^{\prime}$ and $\varphi^{\prime \prime}$ of $\mathfrak{A}$.

Proof. $\|Q\|$ is the l.u.b. of the spectrum of $Q$ and $\left\|Q^{-1}\right\|$ is the l.u.b. of the spectrum of $Q^{-1}$, which is the inverse of the g.l.b. of the spectrum of $Q$. Hence (3.12) follows. Since $\sup _{\varphi} \varphi(Q)=\|Q\|, \inf _{\varphi} \varphi(Q)=\left\|Q^{-1}\right\|^{-1}$ for $Q>0$, we have (3.13).

Lemma 3.8. If $X$ and $Y$ are elements of a Banach algebra, $X^{-1}$ exists and $\|Y\|\left\|X^{-1}\right\|=\delta<1$, then $X+Y$ has an inverse $\left\|(X+Y)^{-1}\right\|$ $\leqq\left\|X^{-1}\right\|(1-\delta)^{-1}$, and

$$
\left\|(X+Y)^{-1}-X^{-1}\right\| \leqq\left\|X^{-1}\right\|\left\{(1-\delta)^{-1}-1\right\} .
$$

Proof. Consider the series

$$
f=X^{-1} \sum_{n=0}^{\infty}\left(-Y X^{-1}\right)^{n}=\left(\sum_{n=0}^{\infty}\left(-X^{-1} Y\right)^{n}\right) X^{-1}
$$

which is absolutely convergent due to $\left\|Y X^{-1}\right\| \leqq\|Y\|\left\|X^{-1}\right\|=\delta<1$. It satisfies $(X+Y) f=f(X+Y)=1$ and hence $f=(X+Y)^{-1}$. Further $\left\|f-X^{-1}\right\|$ $\leqq\left\|X^{-1}\right\| \sum_{n=1}^{\infty} \delta^{n} \leqq\left\|X^{-1}\right\|\left\{(1-\delta)^{-1}-1\right\}$.

Lemma 3.9. Let $Q>0, Q \in \mathfrak{A}$.

(1) $\alpha_{l}(Q) \leqq(\alpha(Q)-1)(\alpha(Q)+1)^{-1}<1$.

(2) $\alpha_{l}(Q) \leqq \alpha_{l^{\prime}}(Q)$ if $l^{\prime} \leqq l$. 
(3) There exists $Q_{(l)} \in \mathfrak{A}([-l, l])$, such that $\left\|Q-Q_{(l)}\right\|\left\|Q_{(l)}^{-1}\right\|=\alpha_{l}(Q)$ and

$$
\left\|Q^{-1}\right\|\left(1-\alpha_{l}(Q)\right) \leqq\left\|Q_{l}^{-1}\right\| \leqq\left\|Q^{-1}\right\|\left(1+\alpha_{l}(Q)\right) .
$$

(4) $\|Q\|_{l} \leqq\left(\|Q\|-\left\|Q^{-1}\right\|^{-1}\right) / 2$.

(5) $\|Q\|_{l} \leqq\|Q\|_{l^{\prime}}$ if $l^{\prime} \leqq l$.

(6) $\alpha_{l}(Q) \leqq\|Q\|_{l}\left\|Q^{-1}\right\|\left(1-\|Q\|_{l}\left\|Q^{-1}\right\|\right)^{-1}$.

(7) $\|Q\|_{l} \leqq\left\|Q^{-1}\right\|^{-1} \alpha_{l}(Q)\left(1-\alpha_{l}(Q)\right)^{-1}$.

(8) $\quad \alpha(\lambda Q)=\alpha(Q), \quad \alpha_{l}(\lambda Q)=\alpha_{l}(Q)$ if $\lambda>0$.

(9) $\|Q+x\|_{l}=\|Q\|_{l}$.

(10) If $\lambda\left\|Q^{-1}\right\|\left(1+\alpha_{l}(Q)\right)<1$, then

$$
\begin{aligned}
& \alpha_{l}(Q-\lambda) \leqq \alpha_{l}(Q)\left(1-\lambda\left\|Q^{-1}\right\|\left(1+\alpha_{l}(Q)\right)\right)^{-1}, \quad \lambda>0 . \\
& \alpha(Q-\lambda) \leqq\left(\alpha(Q)-\lambda\left\|Q^{-1}\right\|\right)\left(1-\lambda\left\|Q^{-1}\right\|\right)^{-1} .
\end{aligned}
$$

Proof. (1) If we set $Q^{(0)}=\left\{\|Q\|+\left\|Q^{-1}\right\|^{-1}\right\} / 2$ then

$$
\begin{aligned}
\alpha_{l}(Q) & \leqq\left\|Q-Q^{(0)}\right\|\left\|\left(Q^{(0)}\right)^{-1}\right\| \\
& =\left(\|Q\|-\left\|Q^{-1}\right\|^{-1}\right) /\left(\|Q\|+\left\|Q^{-1}\right\|^{-1}\right)=(\alpha(Q)-1)(\alpha(Q)+1)^{-1} .
\end{aligned}
$$

(4) follows from $\|Q\|_{l} \leqq\left\|Q-Q^{(0)}\right\|=\left(\|Q\|-\left\|Q^{-1}\right\|^{-1}\right) / 2$. (2) and (5) are obvious. (3). Since $\left\|Q_{l}^{-1}\right\|=\sup \|\psi\|\left\|\pi\left(Q_{l}\right) \psi\right\|^{-1} \geqq\left\|Q_{l}\right\|^{-1}$ where the sup is over all non zero vectors in a faithful representation $\pi$ of $\mathfrak{A}$. Hence as $\left\|Q_{l}\right\| \rightarrow \infty,\left\|Q-Q_{l}\right\|\left\|Q_{l}^{-1}\right\| \geqq\left\|Q_{l}\right\|\left\|Q_{l}^{-1}\right\|\left(1-\|Q\| /\left\|Q_{l}\right\|\right)$ becomes $\geqq 1$. By (3.16), if $\left\|Q-Q_{l}\right\|\left\|Q_{l}^{-1}\right\|<\alpha_{l}(Q)+\varepsilon$ with $\varepsilon<\left(1-\alpha_{l}(Q)\right) / 2$, then $\left\|Q_{l}\right\|$ is bounded by a constant. Hence there exists $Q_{(l)} \in \mathfrak{A}([-l, l])$ such that $\left\|Q-Q_{(l)}\right\|\left\|Q_{(l)}^{-1}\right\|=\alpha_{l}(Q)$ by the compactness of a bounded closed set in $\mathfrak{A}([-l, l])$. We have

$$
\left\|Q Q_{(l)}^{-1}-1\right\| \leqq\left\|Q-Q_{(l)}\right\|\left\|Q_{(l)}^{-1}\right\| \leqq \alpha_{l}(Q) .
$$

Hence $1-\alpha_{l}(Q) \leqq\left\|Q Q_{l}^{-1}\right\| \leqq 1+\alpha_{l}(Q)$ and

$$
\left\|Q_{(l)}^{-1}\right\| \leqq\left\|Q^{-1}\right\|\left\|Q Q_{(l)}^{-1}\right\| \leqq\left\|Q^{-1}\right\|\left(1+\alpha_{l}(Q)\right) .
$$

From (3.28) and Lemma 3.8, $\left\|Q_{(l)} Q^{-1}\right\|=\left\|\left(1+\left(Q Q_{(l)}^{-1}-1\right)\right)^{-1}\right\|$ $\leqq\left(1-\alpha_{l}(Q)\right)^{-1}$. Therefore

$$
\left\|Q_{(l)}^{-1}\right\| \geqq\left\|Q^{-1}\right\|\left\|Q_{(l)} Q^{-1}\right\|^{-1} \geqq\left\|Q^{-1}\right\|\left(1-\alpha_{l}(Q)\right) .
$$

By Lemma 3.8, we have

$$
\left\|\left(Q^{(l)}\right)^{-1}\right\| \leqq\left\|Q^{-1}\right\|\left(1-\left\|Q-Q^{(l)}\right\|\left\|Q^{-1}\right\|\right)^{-1} .
$$


Hence we have

$$
\alpha_{l}(Q) \leqq\left\|Q-Q^{(l)}\right\|\left\|\left(Q^{(l)}\right)^{-1}\right\| \leqq\left\|Q^{-1}\right\|\|Q\|_{l}\left(1-\|Q\|_{l}\left\|Q^{-1}\right\|\right)^{-1}
$$

where $Q^{(l)} \in \mathfrak{A}([-l, l]),\left\|Q-Q^{(l)}\right\|=\|Q\|_{l}$. This proves (6). (7) follows from (3).

(8) and (9) are immediate from definitions. To obtain (10), we note that if $Q_{(l)}$ is given by (3), then, by Lemma 3.8,

$$
\left\|\left(Q_{(l)}-\lambda\right)^{-1}\right\| \leqq\left\|Q_{(l)}^{-1}\right\|\left(1-|\lambda|\left\|Q_{(l)}^{-1}\right\|\right)^{-1}
$$

and hence

$$
\begin{aligned}
& \alpha_{l}(Q-\lambda) \leqq\left\|Q-Q_{(l)}\right\|\left\|\left(Q_{(l)}-\lambda\right)^{-1}\right\| \\
\leqq & \left\|Q-Q_{(l)}\right\|\left\|Q_{(l)}^{-1}\right\|\left(1-\lambda\left\|Q_{(l)}^{-1}\right\|\right)^{-1} \\
\leqq & \alpha_{l}(Q)\left(1-|\lambda|\left\|Q^{-1}\right\|\left(1+\alpha_{l}(Q)\right)\right)^{-1}
\end{aligned}
$$

where we have used (3.29).

Since $\alpha(Q) \geqq\left\|Q^{-1}\right\| Q \geqq 1, \alpha(Q)-\lambda\left\|Q^{-1}\right\| \geqq\left\|Q^{-1}\right\|(Q-\lambda) \geqq 1-\left\|Q^{-1}\right\| \lambda$. Hence we have (3.26).

Lemma 3.10. Let $v$ be a state of $\mathfrak{A}, a>0,1<\alpha<\infty, \lim \alpha_{1}=0$, $\alpha_{l}>0$. Let $\Sigma$ be the set of $Q \in \mathfrak{U}, Q \geqq 0$ such that $v(Q)=a, \alpha(Q) \leqq \alpha,\|Q\|_{l}\left\|Q^{-1}\right\|$ $\leqq \alpha_{l}, l=N, N+1, \ldots$. Then $\Sigma$ is a convex, compact subset of $\mathfrak{U}$.

Proof. First we prove the convexity. Let $Q=\lambda Q_{1}+(1-\lambda) Q_{2}, Q_{1} \in \Sigma$, $Q_{2} \in \Sigma, 0 \leqq \lambda \leqq 1 . v(Q)=\lambda v\left(Q_{1}\right)+(1-\lambda) v\left(Q_{2}\right)=a$. Since $Q_{1} \geqq 0, Q_{2} \geqq 0$, we have $Q \geqq 0$.

$$
\begin{aligned}
& \|Q\| \leqq \lambda\left\|Q_{1}\right\|+(1-\lambda)\left\|Q_{2}\right\| \leqq \alpha\left\{\lambda\left\|Q_{1}^{-1}\right\|^{-1}+(1-\lambda)\left\|Q_{2}^{-1}\right\|^{-1}\right\} . \\
& \left\|Q^{-1}\right\|^{-1}=\inf _{\varphi} \varphi(Q) \geqq \lambda \inf \varphi\left(Q_{1}\right) \\
& \quad+(1-\lambda) \inf \varphi\left(Q_{2}\right) \geqq \lambda\left\|Q_{1}^{-1}\right\|^{-1}+(1-\lambda)\left\|Q_{2}^{-1}\right\|^{-1} .
\end{aligned}
$$

Hence $\alpha(Q) \leqq \alpha$. Similarly,

$$
\|Q\|_{n} \leqq \lambda\left\|Q_{1}\right\|_{n}+(1-\lambda)\left\|Q_{2}\right\|_{n} \leqq \alpha_{n}\left(\lambda\left\|Q_{1}^{-1}\right\|^{-1}+(1-\lambda)\left\|Q_{2}^{-1}\right\|^{-1}\right) .
$$

Hence $\|Q\|_{l}\left\|Q^{-1}\right\| \leqq \alpha_{l}$.

Next we prove the compactness. From $v(Q)=a \geqq\left\|Q^{-1}\right\|^{-1} \geqq \alpha^{-1}\|Q\|$, we have $\|Q\| \leqq a \alpha$. Similarly $\|Q\|_{l} \leqq \alpha_{l}\left\|Q^{-1}\right\|^{-1}=\alpha_{l} a$. Therefore $\Sigma$ is a subset of a compact set. We now prove that $\Sigma$ is closed. Let $Q_{n} \in \Sigma$, $\lim _{n \rightarrow \infty}\left\|Q-Q_{n}\right\|=0$. We have $v(Q)=\lim v\left(Q_{n}\right)=\alpha$. From $Q_{n} \geqq\left\|Q_{n}^{-1}\right\|^{-1}$ $\geqq \alpha^{-1}\left\|Q_{n}\right\| \geqq \alpha^{-1} a$, we have $Q \geqq \alpha^{-1} a$. Hence by Lemma $3.8, \lim _{n \rightarrow \infty}\left\|Q_{n}^{-1}\right\|$ $=\left\|Q^{-1}\right\|$. Hence $\lim _{n \rightarrow \infty} \alpha\left(Q_{n}\right)=\alpha(Q) \leqq \alpha, \lim _{n \rightarrow \infty}\left\|Q_{n}\right\|_{l}\left\|Q_{n}^{-1}\right\|=\|Q\|_{l}\left\|Q^{-1}\right\|$ $\leqq \alpha_{l}$. Q.E.D. 
Corollary 3.11. Let $v$ be a state of $\mathfrak{A}, x>1,1<\alpha<\infty, a>0$. The set of $Q \in \mathfrak{A}, Q \geqq 0$ such that $v(Q)=a,\|Q\|_{M, x}\left\|Q^{-1}\right\| \leqq \alpha$ is a convex compact subset of $\mathfrak{A}$.

Proof. From $\|Q\|_{M, x}\left\|Q^{-1}\right\| \leqq \alpha$, we have $\alpha(Q) \leqq \alpha,\|Q\|_{l}\left\|Q^{-1}\right\| \leqq x^{-l} \alpha$, $l=M, M+1, \ldots$ The set is closed and hence compact. From (3.34) (3.36), $\left\|\lambda_{1} Q_{1}+\lambda_{2} Q_{2}\right\|_{M, x}\left\|\left(\lambda_{1} Q_{1}+\lambda_{2} Q_{2}\right)^{-1}\right\| \leqq a$ if $Q_{1}$ and $Q_{2}$ are in the set, $\lambda_{1}+\lambda_{2}=1, \lambda_{1} \geqq 0, \lambda_{2} \geqq 0$. Hence it is convex.

Corollary 3.12. The set of $Q \in \mathfrak{A}, Q \geqq 0$ such that $v(Q)=a, \alpha(Q) \leqq \alpha$, $\alpha_{l}(Q) \leqq \alpha_{l}, l=N, N+1, \ldots$ is a subset of a compact convex set, if $\lim \alpha_{l}=0$.

Proof. This follows from (3.22) and Lemma 3.10.

Remark. In [12], quantities of the form $\beta_{l}(Q)=\sup _{\varphi \varphi^{\prime} \varphi^{\prime \prime}} \varphi \otimes \varphi^{\prime}(Q) / \varphi \otimes \varphi^{\prime \prime}(Q)$ has been used instead of our $\alpha_{l}(Q)$, where $\varphi$ runs over states of $\mathfrak{A}([-n, n])$ and $\varphi^{\prime}, \varphi^{\prime \prime}$ runs over states of $\mathfrak{A}(Z \backslash[-n, n]) . \quad \beta_{l}\left(\lambda Q_{1}+(1-\lambda) Q_{2}\right)$ $\leqq \max \left\{\beta_{l}\left(Q_{1}\right), \beta_{l}\left(Q_{2}\right)\right\}$ for $Q_{1}, Q_{2} \geqq 0,0 \leqq \lambda \leqq 1$. Hence the condition $\beta_{l}(Q) \leqq \beta_{l}$ is stable under convex combination of $Q . \mathfrak{A}_{1}$ is a Fréchet Montel space.

\section{§ 4. Time Translation}

For any two elements $Q$ and $R$ in $\mathfrak{A}$, define

$$
\begin{aligned}
\delta(R) Q & =[R, Q], \\
\{\exp \delta(R)\} Q & =\sum_{n=0}^{\infty}(n !)^{-1} \delta(R)^{n} Q .
\end{aligned}
$$

We extend this definition to $R=H(I)$

$$
\delta(H(I)) Q=\sum_{j:[j, j+r] \subset I}\left[\tau_{s}(j) \Phi, Q\right] .
$$

If $Q \in \mathfrak{A}_{0}$, the sum terminates at finite $j$.

Lemma 4.1. Let $C_{l}^{m}(n)$ be numbers such that

$$
\begin{gathered}
C_{l}^{m+1}(n)=(l-r+1) C_{l}^{m}(n)+2 \sum_{k=1}^{r} C_{l-k}^{m}(n) \\
C_{l}^{0}(n)=\delta_{l n}(\text { i.e. }=1 \quad \text { if } \quad l=n,=0 \quad \text { if } \quad l \neq n)
\end{gathered}
$$

where $l, m \in Z, m \geqq 0, n \geqq r-1$. Then $C_{l}^{m}(n) \geqq 0$, and

$$
C_{l}^{m}(n)=0 \text { if } l<n \text { or } l>n+m r .
$$

Let

$$
f_{n}(x, y)=\sum_{m, l} C_{l}^{m}(n) x^{l-n} y^{m r+n-l} / m !
$$


Then, (4.7) is absolutely convergent for all $x$ and $y$, and is given by

$$
f_{n}(x, y)=\exp \left[(n-r+1) y^{r}+2 \sum_{k=1}^{r} k^{-1}(x / y)^{k}\left\{\exp k y^{r}-1\right\}\right] .
$$

Let

$$
\begin{aligned}
F_{n}(x) & =\sum_{m, l} C_{l}^{m}(n) x^{m} / m !, \\
F_{n}^{L}(x) & =\sum_{m} \sum_{l>L} C_{l}^{m}(n) x^{m} / m ! . \quad(L \geqq n .)
\end{aligned}
$$

Then

$$
\begin{gathered}
F_{n}(x)=\exp \left[(n-r+1) x+2 \sum_{k=1}^{r} k^{-1}\{\exp k x-1\}\right], \\
F_{n}^{L r+n}(x) \leqq(L+1) !^{-1}\left[2 \sum_{k=1}^{r} k^{-1}\left(e^{k x}-1\right)\right]^{L+1} F_{n}(x) \quad(x>0),
\end{gathered}
$$

where $L \geqq 0$.

Proof. $C_{l}^{m}(n) \geqq 0$ and (4.6) are immediate from (4.4) and (4.5) by induction on $m$. By (4.4) and (4.6), we have

$$
\sup _{l}\left|C_{l}^{m+1}(n)\right| \leqq\{(n+m r)-r+1+2 r\} \sup _{l}\left|C_{l}^{m}(n)\right| .
$$

By repetition, we have $\sup _{l}\left|C_{l}^{m}(n)\right| \leqq \prod_{k=1}^{m}(n+1+k r)$. Therefore (4.7) is absolutely convergent near $x=y=0$ and defines a holomorphic function.

Due to (4.4), $f_{n}(x, y)$ satisfies within the polycircle of convergence the following partial differential equation

$$
\begin{aligned}
\left(r y^{r}\right)^{-1}[x(\partial / \partial x)+y(\partial / \partial y)] f_{n} & \\
& =\{x(\partial / \partial x)+n-r+1\} f_{n}+2 \sum_{k=1}^{r}(x / y)^{k} f_{n} .
\end{aligned}
$$

From (4.5), we have the initial condition

$$
f(0,0)=1 .
$$

After the change of variables

$s=\log x, \quad t=\log y-y^{r}, \quad u=(s+t) / 2, \quad v=(s-t) / 2$,

we have

$$
\begin{gathered}
(\partial / \partial u) f_{n}(x, y)=g(x / y)\left(\partial y^{r} / \partial u\right) f_{n}(x, y), \\
g(x / y)=(n-r+1)+2 \sum_{k=1}^{r}(x / y)^{k},
\end{gathered}
$$


where $(\partial / \partial u)$ is for fixed $v$. Since $x / y=\exp \left(2 v-y^{r}\right)$, we have

$$
\begin{aligned}
\log f_{n}(x, y)+k(v) & =\int g\left(\exp \left[2 v-y^{r}\right]\right) \mathrm{d}\left(y^{r}\right) \\
& =(n-r+1) y^{r}-2 \sum_{k=1}^{r} k^{-1}(x / y)^{k},
\end{aligned}
$$

where the unknown function $k(v)$ can be determined from (4.15) by taking the limit $y \rightarrow 0$ with $x / y$ fixed at $e^{2 \varrho}$ :

$$
k(\varrho)=-2 \sum_{k=1}^{r} k^{-1} e^{2 k \varrho} .
$$

Therefore we have (4.8). By definition, $F_{n}(x)=f_{n}\left(x^{1 / r}, x^{1 / r}\right)$ and we have (4.11).

To obtain (4.12), we consider

$$
\begin{aligned}
\hat{f_{n}}(x, y) & =\exp \left[(n-r+1) y^{r}+2(x / y)^{r} \sum_{k=1}^{r} k^{-1}\left\{\exp k y^{r}-1\right\}\right] \\
& =\sum_{m, l} \hat{C}_{l}^{m}(n) x^{l-n} y^{m r+n-l} / m !
\end{aligned}
$$

By the power series expansion of exponentials in (4.11) as well as in (4.21), we obtain expressions for $C_{l}^{m}(n)$ and $\hat{C}_{l}^{m}(n)$ as sums of positive terms. The change of $(x / y)^{k}$ in (4.11) to $(x / y)^{r}=(x / y)^{k}(x / y)^{r-k}$ increases the power of $x$ by $r-k \geqq 0$ while keeping the total degree in $x$ and $y$ as well as the numerical coefficient of each term. Therefore all terms in the expression for $C_{l}^{m}(n)$ moves into expressions for $\hat{C}_{l^{\prime}}^{m}(n)$ with the same $m$ and higher $l^{\prime} \geqq l$. Hence

$$
\begin{gathered}
0 \leqq \sum_{l>L} C_{l}^{m}(n) \leqq \sum_{l>L} \hat{C}_{l}^{m}(n) \\
0 \leqq F_{n}^{L}(x) \leqq \hat{F}_{n}^{L}(x) \equiv \sum_{n} \sum_{l>L} \hat{C}_{l}^{m}(n) x^{m} / m !, \quad(x>0) .
\end{gathered}
$$

By Tailor's expansion theorem, we have

$$
\begin{aligned}
\hat{F}_{n}^{L r+n}(x) & =\left\{\varphi_{n}(x, x)-\sum_{k=0}^{L}\left(x^{k} / k !\right)(\partial / \partial \xi)^{k} \varphi_{n}(\xi, x)\right\}_{\xi=0} \\
& =\left.(L+1) !^{-1} x^{L+1}(\partial / \partial \xi)^{L+1} \varphi_{n}(\xi, x)\right|_{\xi=\theta x}
\end{aligned}
$$

where $0<\theta<1$ and

$$
\begin{aligned}
\varphi_{n}(\xi, \eta) & =\hat{f}_{n}\left(\xi^{1 / r}, \eta^{1 / r}\right) \\
& =\exp \left[(n-r+1) \eta+2 \sum_{k=1}^{r} k^{-1}\left(e^{k \eta}-1\right)(\xi / \eta)\right] .
\end{aligned}
$$

The main point of introducing $\hat{F}_{n}$ is that (4.24) is easier to calculate for $\hat{F}$ than for $F$. From (4.23), (4.24) and (4.25), we obtain (4.12). 
Theorem 4.2. (i) $\{\exp \delta(\beta H(I))\} Q$ converges absolutely in the norm for any complex $\beta ; Q \in \mathfrak{A}_{1}$, and $I \subset Z$. (ii) $\exp \delta(\beta H(I))$ is a group of automorphisms of $\mathfrak{A}\left(I^{\prime}\right) \cap \mathfrak{U}_{1}$ and $\mathfrak{U}\left(I^{\prime}\right) \cap \mathfrak{U}_{2}$ with one complex parameter $\beta$ for any $I^{\prime} \supset I$. $\{\exp \delta(\beta H(I))\} Q$ is analytic in $\beta$ for each $Q \in \mathfrak{U}_{1}$. (iii) $\tau_{T}^{I}(t)=\exp \delta($ it $H(I))$ for real $t$ has a unique extention to a continuous one parameter group of ${ }^{*}$-automorphisms of $\mathfrak{A}$, commuting with $\tau_{s}(a)$. $\tau_{T}^{Z}=\tau_{T}$

(iv) If $Q \in \mathfrak{U}([0, n])$, then for any $N \geqq 0$

$$
\begin{gathered}
\{\exp \delta(\beta H(I))\} Q=Q_{N, I}(\beta)+\delta Q_{N, I}(\beta), \\
Q_{N, I}(\beta) \equiv \exp \delta[\beta H(I \cap[-N, n+N])] Q \in \mathfrak{A}([-N, n+N]), \\
\left\|\delta Q_{N, I}(\beta)\right\| \leqq(1+[N / r]) !^{-1} \alpha(\beta)^{[N / r]+1} F_{n}(2|\beta|\|\Phi\|)\|Q\|, \\
\alpha(\beta)=2 \sum_{k=1}^{r} k^{-1}(\exp 2 k|\beta|\|\Phi\|-1),
\end{gathered}
$$

where $[N / r]$ denotes the largest integer not exceeding $N / r$, and $n \geqq r-1$.

Proof. By definition, if $Q \in \mathfrak{U}([0, n])$

$$
\delta(\beta H(Z))^{m} Q=\beta^{m} \Sigma\left[\tau_{s}\left(j_{m}\right) \Phi,\left[\ldots,\left[\tau_{s}\left(j_{1}\right) \Phi, Q\right] \ldots\right]\right],
$$

where the sum is over all $j_{1} \ldots j_{m} \in Z$ such that $\left[j_{k}, j_{k}+r\right]$ has a non empty intersection with the interval

$$
I\left(j_{1} \ldots j_{k-1}\right)=[0, n] \cup\left(\bigcup_{l<k}\left[j_{l}, j_{l}+r\right]\right)
$$

for each $k=1,2, \ldots m$. Let $C_{l}^{m}(n)$ be the number of terms in (4.30) for which the length of the interval $I\left(j_{1} \ldots j_{m}\right)$ is $l$. It satisfies (4.4) and (4.5), where the first term of (4.4) represents the case in which $\left[j_{m+1}, j_{m+1}+r\right]$ falls in $I\left(j_{1} \ldots j_{m}\right)$ and the rest represents cases in which $\left[j_{m+1}, j_{m+1}+r\right]$ has still non empty intersection with $I\left(j_{1} \ldots j_{m}\right)$ and sticks out to either side of $I\left(j_{1} \ldots j_{m}\right)$. We now have two inequalities

$$
\begin{aligned}
\left\|\delta(\beta H(I))^{m} Q\right\| & \leqq(2|\beta|\|\Phi\|)^{m}\|Q\| \sum_{l} C_{l}^{m}(n), \\
\left\|\delta(\beta H(I))^{m} Q-\delta(\beta H(I \cap[-N, n+N]))^{m} Q\right\| & \leqq(2|\beta|\|\Phi\|)^{m}\|Q\| \sum_{l>N+n} C_{l}^{m}(n) .
\end{aligned}
$$

Note that the change of $Z$ to $I \subset Z$ only decreases the number of terms in (4.30).

We are now ready to prove (i) and (iv). If we write $Q=\sum_{k=k_{0}}^{\infty} Q_{k}$, $Q_{k}=Q^{(k)}-Q^{(k-1)} \in \mathfrak{A}([-k, k]),\left\|Q-Q^{(k)}\right\|=\|Q\|_{k}\left(k \geqq k_{0}\right), Q^{\left(k_{0}-1\right)}=0$, we have $\left\|Q_{k}\right\| \leqq\|Q\|_{k}+\|Q\|_{k-1}$ where $\|Q\|_{k_{0}-1}$ is to be replaced by $\|Q\|$. 
Hence if $Q \in \mathfrak{A}_{1}$, then $\Sigma x^{k}\left\|Q_{k}\right\|<\infty$ for any $x>0$. From our discussion, we have

$$
\begin{aligned}
& \sum_{m=0}^{\infty}(m !)^{-1}|\beta|^{m} \sum_{j_{1} \ldots j_{m}}\left\|\left[\tau_{s}\left(j_{m}\right) \Phi,\left[\ldots,\left[\tau_{s}\left(j_{1}\right) \Phi, Q\right] \ldots\right]\right]\right\| \\
\leqq & \sum_{k=k_{0}}^{\infty} \sum_{m=0}^{\infty}(m !)^{-1}(2|\beta|\|\Phi\|)^{m} \sum_{l} C_{l}^{m}(2 k)\left(\|Q\|_{k}+\|Q\|_{k-1}\right) \\
\leqq & \sum_{k=k_{0}}^{\infty} F_{2 k}(2|\beta|\|\Phi\|)\left(\|Q\|_{k}+\|Q\|_{k-1}\right) .
\end{aligned}
$$

By Lemma 4.1, $F_{2 k}(x)=\left(e^{2 x}\right)^{k} F_{0}(x)$. Hence (4.34) is finite and we have (i). Similarly, for $Q \in \mathfrak{A}[0, n]$,

$$
\begin{aligned}
\left\|\delta Q_{N, I}(\beta)\right\| & \leqq \sum_{m=0}^{\infty}(m !)^{-1}(2|\beta|\|\Phi\|)^{m} \sum_{l>N+n} C_{l}^{m}(n)\|Q\| \\
& \leqq F_{n}^{N+n}(2|\beta|\|\Phi\|)\|Q\| \\
& \leqq F_{n}^{[N / r] r+n}(2|\beta|\|\Phi\|)\|Q\| .
\end{aligned}
$$

By substituting (4.12) into (4.35), we obtain (4.28). selves.

To obtain (ii), we first show that $\mathfrak{H}_{1}$ and $\mathfrak{H}_{2}$ are mapped into them-

Let $Q=\Sigma Q_{k}, Q_{k} \in \mathfrak{U}([-k, k]),\left\|Q_{k}\right\| \leqq\|Q\|_{k}+\|Q\|_{k-1}, k=k_{0}, k_{0}+1, \ldots$ where $\|Q\|_{k_{0}-1}$ is to be replaced by $\|Q\|$. We have for $n \geqq k_{0}$

$$
\begin{aligned}
\gamma_{n}=\|\exp \delta(\beta H(I)) Q\|_{n} \\
\leqq \sum_{k=k_{0}}^{n} F_{2 k}^{(n-k)+2 k}(2\|\beta \Phi\|)\left\|Q_{k}\right\|+\sum_{k=n+1}^{\infty} F_{2 k}(2\|\beta \Phi\|)\left\|Q_{k}\right\| .
\end{aligned}
$$

Hence, for $x>1$,

$$
\begin{aligned}
& \sum_{n=k_{0}}^{\infty} x^{n} \gamma_{n} \\
& \leqq \\
& \left.\qquad \sum_{N=0}^{\infty} x^{N}(1+[N / r]) !^{-1} \alpha(\beta)^{[N / r]+1}\right)\left(\sum_{k=k_{0}}^{\infty}\left(x e^{4\|\beta \Phi\|}\right)^{k}\left\|Q_{k}\right\|\right) F_{0}(2\|\beta \Phi\|) \\
& \quad+\sum_{k>k_{0}}\left\{x e^{4\|\beta \Phi\|}\right\}^{k}\left\|Q_{k}\right\|(x-1)^{-1} F_{0}(2\|\gamma \Phi\|),
\end{aligned}
$$

which is finite if $Q \in \mathfrak{A}_{1}$. Next, if $Q \in \mathfrak{A}_{2}$, we have

$$
\begin{aligned}
\log \gamma_{n} & \left.\leqq \log \sum_{k=k_{0}}^{n+1} \gamma_{n k} \leqq \log \left\{n+2-k_{0}\right) \max \gamma_{n k}\right\} \\
& =\log \left(n+2-k_{0}\right)+\max \log \gamma_{n k}
\end{aligned}
$$


where $\gamma_{n k}=\delta_{n-k}^{1} \delta_{k}^{2}$ for $k=k_{0} \ldots n$,

$$
\begin{aligned}
& \delta_{N}^{1}=(1+[N / r]) !^{-1} \alpha(\beta)^{[N / r]+1}, \\
& \delta_{k}^{2}=F_{2 k}(2\|\beta \Phi\|)\left\|Q_{k}\right\|,
\end{aligned}
$$

$\gamma_{n, n+1}=\sum_{k=n+1}^{\infty} F_{2 k}(2\|\beta \Phi\|)\left\|Q_{k}\right\| \leqq\left(1-e^{-1}\right)^{-1} F_{2 n}(2\|\beta \Phi\|) \gamma_{n, n+1}^{\prime}$,

$\gamma_{n, n+1}^{\prime}=\sup _{k \geqq 1}\left[e^{(1+4\|\beta \Phi\|)}\right]^{k}\left\|Q_{n+k}\right\|$.

We have, for $k_{0} \leqq k \leqq n$ and $n \geqq k_{0}$,

$n^{-1}[(n / r) \log n-(k / r) \log k-[(n-k) / r] \log (n-k)] \leqq r^{-1} \log 2$,

$n^{-1} \log F_{2 k}(2\|\beta \Phi\|) \leqq 4\|\beta \Phi\|+\log F_{0}(2\|\beta \Phi\|)$,

$n^{-1}\left\{\log \delta_{n-k}^{1}+((n-k) / r) \log (n-k)\right\}$

$\leqq \sup _{N \geqq 1} N^{-1}\{(N / r) \log N-\log \Gamma(N / r+1)\}+(1+1 / r) \log \alpha(\beta)<\infty$,

$n^{-1}\left\{\log \left\|Q_{k}\right\|+(k / r) \log k\right\} \leqq \sup _{l \geqq 1} l^{-1}\left\{\log \left\|Q_{l}\right\|+(l / r) \log l\right\}<\infty$,

$n^{-1}\left\{\log \gamma_{n, n+1}^{\prime}+(n / r) \log n\right\}$

$$
\begin{aligned}
\leqq & \sup _{k, n \geq 1}\left\{(1+(k / n)) \sup _{l}\left\{l^{-1}\left(\log \left\|Q_{l}\right\|+(l / r) \log l\right)\right\}\right. \\
& \left.\left.-r^{-1} \log (n+k)\right]+r^{-1} \log n+(k / n)(1+4\|\beta \Phi\|)\right\}<\infty .
\end{aligned}
$$

Therefore $\sup _{n} n^{-1}\left(\gamma_{n}+(n / r) \log n\right)<\infty$.

The isomorphism property of $\exp \delta(\beta H(I))$ follows from the Leibnitz formula:

$\delta(\beta H(I))^{m}\left(Q_{1} Q_{2}\right)=\sum_{k=0}^{m}\left(\begin{array}{c}m \\ k\end{array}\right)\left(\delta(\beta H(I))^{k} Q_{1}\right)\left(\delta(\beta H(I))^{m-k} Q_{2}\right)$.

To see the group property, we first note that

$$
\sum_{m, n}\left\|\delta\left(\beta_{1} H(I)\right)^{m} \delta\left(\beta_{2} H(I)\right)^{n} Q / m ! n !\right\|<\infty .
$$

Hence we can change the order of summation to obtain

$$
\exp \delta\left(\beta_{1} H(I)\right) \exp \delta\left(\beta_{2} H(I)\right)=\exp \delta\left(\left(\beta_{1}+\beta_{2}\right) H(I)\right) .
$$

Since $\exp \delta(\beta H(I)) Q$ has a power series expansion in $\beta$ which converges absolutely for all $\beta$, it is analytic in $\beta$. This completes the proof of (ii). The $*$-isomorphism property of $\tau_{T}^{I}(t)$ follows from

$$
[\delta(\text { it } H(I)) Q]^{*}=\delta(\text { it } H(I)) Q .
$$


Any $*$-isomorphism of $\mathfrak{A}_{0}$ into $\mathfrak{A}$ can be uniquely extended to a $*$-isomorphism of $\mathfrak{A}$ into $\mathfrak{A}$. The uniqueness guarantees that the extension to $\mathfrak{A}$ of the restriction to $\mathfrak{A}_{0}$ of $\exp \delta(i t H(I))$ is the extension of $\exp \delta(i t H(I))$. The group property and the continuity in $\beta$ is preserved in the extension. Hence each $\exp \delta(i t H(I))$ has an inverse $\exp \delta(-i t H(I))$ and therefore must be a $*$-automorphism. The commutativity with $\tau_{s}(a)$ is immediate. This proves (iii). Q.E.D.

Corollary 4.3. If $|t| \leqq(2 r\|\Phi\|)^{-1} \log |a|$, then

$$
\lim _{a \rightarrow \infty} e^{|a| \varrho}\left\|\left[Q_{1}, \tau_{s}(a) \tau_{T}(t) Q_{2}\right]\right\|=0
$$

for any $Q_{1}, Q_{2} \in \mathfrak{A}_{0}$ and $\varrho>0$.

The proof is immediate from Theorem 4.2 (iv).

Remark 4.4. The convergence of $\exp \delta\left(\beta H\left(Z^{v}\right)\right) Q$ for $v$ dimensional lattice has been proved for $|\beta|<[2 r(r-1)\|\Phi\|]^{-1}$. ([11, 13].) In this case, a weaker commutativity can be proven in a region where $|t|$ can grow to infinity as $|a| \rightarrow \infty$.

\section{§ 5. Expansionals}

Definition 5.1. (cf. [5]). Let $Q \in \mathfrak{A}_{0}, Q(\beta ; I)=\exp \delta(\beta H(I)) Q$. Then

$$
\begin{aligned}
& E_{r}(Q ; H(I))=\sum_{n=0}^{\infty} \int_{0}^{1} \mathrm{~d} \beta_{1} \int_{0}^{\beta_{1}} \mathrm{~d} \beta_{2} \ldots \int_{0}^{\beta_{n-1}} \mathrm{~d} \beta_{n} \prod_{j}^{n \rightarrow 1} Q\left(\beta_{j} ; I\right), \\
& E_{l}(Q ; H(I))=\sum_{n=0}^{\infty} \int_{0}^{1} \mathrm{~d} \beta_{1} \int_{0}^{\beta_{1}} \mathrm{~d} \beta_{2} \ldots \int_{0}^{\beta_{n-1}} \mathrm{~d} \beta_{n} \prod_{j}^{1 \rightarrow n} Q\left(-\beta_{j} ; I\right),
\end{aligned}
$$

where

$$
\prod_{j}^{1 \rightarrow n} A_{j}=A_{1} \ldots A_{n}, \quad \prod_{j}^{n \rightarrow 1} A_{j}=A_{n} \ldots A_{1} .
$$

By a change of integration variables, we also have, for real $\beta$,

$$
\begin{aligned}
& E_{r}(\beta Q ; \beta H(I))=\sum_{n=0}^{\infty} \int_{0}^{\beta} \mathrm{d} \beta_{1} \int_{0}^{\beta_{1}} \mathrm{~d} \beta_{2} \ldots \int_{0}^{\beta_{n-1}} \mathrm{~d} \beta_{n} \prod_{j}^{n \rightarrow 1} Q\left(\beta_{j} ; I\right), \\
& E_{l}(\beta Q ; \beta H(I))=\sum_{n=0}^{\infty} \int_{0}^{\beta} \mathrm{d} \beta_{1} \int_{0}^{\beta_{1}} \mathrm{~d} \beta_{2} \ldots \int_{0}^{\beta n_{-1}} \mathrm{~d} \beta_{n} \prod_{j}^{1 \rightarrow n} Q\left(-\beta_{j} ; I\right) .
\end{aligned}
$$

For $Q \in \mathfrak{A}([0, n])$, the sums and integrals are absolutely and uniformly (for bounded $\|\beta \Phi\|,\|Q\|$ and $n$ ) convergent because $\left\|Q\left(\beta_{j} ; I\right)\right\|$ 
$\leqq F_{n}(2\|\beta \Phi\|)\|Q\|$ for $\left|\beta_{j}\right| \leqq|\beta|$ and hence

$$
\begin{aligned}
& \sum_{n=0}^{\infty} \int_{0}^{\beta} \mathrm{d} \beta_{1} \int_{0}^{\beta_{1}} \mathrm{~d} \beta_{2} \ldots \int_{0}^{\beta_{n-1}} \mathrm{~d} \beta_{n} \prod_{j=1}^{n}\left\|Q\left(\beta_{j} ; I\right)\right\| \\
& \leqq \\
& \exp \left\{|\beta| F_{n}(2\|\beta \Phi\|)\|Q\|\right\}<\infty .
\end{aligned}
$$

For bounded operators $Q$ and $R$,

$$
\begin{gathered}
\{\exp \delta(\beta R)\} Q=e^{\beta R} Q e^{-\beta R}, \\
E_{r}(\beta Q ; \beta R)\{\exp \beta R\}=\exp \beta(Q+R), \\
\{\exp \beta R\} E_{l}(\beta Q ; \beta R)=\exp \beta(Q+R) .
\end{gathered}
$$

These formulas can be easily proved by noting that they are 1 for $\beta=0$ and each side of 3 equations satisfy differential equations $(\mathrm{d} / \mathrm{d} \beta) S=[R, S]$; $(\mathrm{d} / \mathrm{d} \beta) S=S(Q+R)$, and $(\mathrm{d} / \mathrm{d} \beta) S=(Q+R) S$, respectively. ((5.7) is used in the proof of (5.8) and (5.9).)

From (5.8) and (5.9), the following formulas follow immediately.

$$
E_{r}\left(Q_{1}+Q_{2} ; R\right)=E_{r}\left(Q_{1} ; Q_{2}+R\right) E_{r}\left(Q_{2} ; R\right),
$$

$$
\begin{gathered}
E_{r}(Q ; R)=E_{l}(Q ;-R-Q), \quad E_{l}(Q ; R)=E_{r}(Q ;-Q-R), \\
E_{r}(Q ; R) E_{l}(-Q ;-R)=E_{l}(-Q ;-R) E_{r}(Q ; R)=1, \\
E_{r}(Q ; R)\{\exp \delta(R)\} Q^{\prime}=\left[\{\exp \delta(Q+R)\} Q^{\prime}\right] E_{r}(Q ; R),
\end{gathered}
$$

$\left[\{\exp \delta(-R)\} Q^{\prime}\right] E_{l}(Q ; R)=E_{l}(Q ; R)\{\exp \delta(-R-Q)\} Q^{\prime}$.

Lemma 5.2. (i) If $Q \in \mathfrak{A}([0, n])$, then

$$
\begin{gathered}
E_{r}(Q ; \lambda H(I))=E_{r}[Q ; \lambda H(I \cap[-N, n+N])]+\delta_{r}(I, N), \\
\left\|\delta_{r}(I, N)\right\| \leqq C_{n} \delta_{N}(\lambda \Phi), \\
\delta_{N}(\lambda \Phi)=(1+[N / r]) !^{-1} \alpha(\lambda)^{[N / r]+1},
\end{gathered}
$$

where $C_{n}$ depends on $n,\|\lambda \Phi\|$ and $\|Q\|$ but is independent of $N$ and $I$.

(ii) The same equation holds when the suffix $r$ is replaced by $l$.

(iii) If $Q \in \mathfrak{A}_{0}$, then $E_{\mathbf{r}}(Q ; \lambda H(I)) \in \mathfrak{A}_{2}, E_{l}(Q ; \lambda H(I)) \in \mathfrak{A}_{2}$.

(iv) Formulas (5.10) (5.14) hold when $R$ is replaced by $\lambda H(I)$ and if $Q_{1}, Q_{2}, Q, Q^{\prime} \in \mathfrak{A}_{0}$.

Proof. We have $\left\|Q\left(\beta^{\prime} ; I\right)\right\| \leqq F_{n}(2\|\lambda \Phi\|)\|Q\|$ and $\left\|Q_{N, I}\left(\beta^{\prime}\right)\right\|$ $\leqq F_{n}(2\|\lambda \Phi\|)\|Q\|$ for $\left|\beta^{\prime}\right| \leqq 1$. Since

$$
\begin{aligned}
\prod_{j}^{n \rightarrow 1} Q\left(\beta_{j} ; I\right)= & \sum_{j=1}^{n}\left(\prod_{k}^{n \rightarrow j+1} Q\left(\beta_{k} ; I\right)\right) \delta Q_{N, I}\left(\beta_{j}\right)\left(\prod_{k}^{j-1 \rightarrow 1} Q_{N, I}\left(\beta_{k}\right)\right) \\
& +\prod_{j}^{n \rightarrow 1} Q_{N, I}\left(\beta_{j}\right)
\end{aligned}
$$


we have (5.16) where, for $n \geqq r-1$,

$$
C_{n}=F_{n}(2\|\lambda \Phi\|)\|Q\| \exp \left\{F_{n}(2\|\lambda \Phi\|)\|Q\|\right\} .
$$

(ii) follows in exactly the same manner. By the Sterling formula, we obtain (iii). The formulas $(5.10) \sim(5.14)$ hold when $R$ is replaced by $\lambda H(I \cap[-N, n+N])$. By taking the limit $N \rightarrow \infty$, we obtain $(5.10) \sim(5.14)$ for $R=\lambda H(I)$. Q.E.D.

\section{§ 6. The Mapping $\mathscr{L}$}

Definition 6.1. Let $I$ be a finite subset of $Z, \varphi$ be a state of $\mathfrak{A}(I)$, $Q=\Sigma u_{l} Q_{l}, u_{l} \in \mathfrak{A}(I), Q_{l} \in \mathfrak{A}(Z \backslash I)$. Then $\varphi(Q) \equiv \Sigma \varphi\left(u_{l}\right) Q_{l} \in \mathfrak{U}(Z \backslash I) \subset \mathfrak{A}$.

It is easily proved that $\varphi(Q)$ does not depend on a particular decomposition $Q=\sum u_{l} Q_{l}$.

Definition 6.2. Let $Q \in \mathfrak{U}$.

$$
\begin{gathered}
\mathscr{L}(Q)=\tau_{c}(1) d^{-2} \operatorname{tr}_{[0,1]}\left(K^{*} Q K\right), \\
K=K_{+} K_{-}, \\
K_{+}=E_{r}\left[-(1 / 2) \tau_{s}(1) \Phi ;-(1 / 2) H(2, \infty)\right], \\
K_{-}=E_{r}\left[-(1 / 2) \tau_{s}(-r) \Phi ;-(1 / 2) H(-\infty,-1)\right] .
\end{gathered}
$$

Lemma 6.3. (i) If $Q \in \mathfrak{U}_{1}$, then $\mathscr{L} Q \in \mathfrak{U}_{1}$. If $Q \in \mathfrak{U}_{2}$, then $\mathscr{L} Q \in \mathfrak{U}_{2}$. (ii) If $Q \geqq 0$, then $\mathscr{L} Q \geqq 0$. If $Q \geqq 0$ and $Q \neq 0$, then $\mathscr{L} Q \neq 0$. If $Q \geqq 0$ and $Q^{-1}$ exists, then $(\mathscr{L} Q)^{-1} \in \mathfrak{A}$. (iii) If $n>r$, then

$$
\begin{gathered}
\mathscr{L}^{n} Q=p_{n} \tau_{c}(n) \varphi_{n}\left(K_{n}^{*} Q K_{n}\right), \\
K_{n}=K_{n+} K_{n-}, K_{n+} \in \mathfrak{U}([1, \infty)), K_{n-} \in \mathfrak{U}((-\infty, 0]) \\
K_{n+}=E_{r}\left(-(1 / 2) \tau_{s}(n) \Psi ;-(1 / 2)\{H(1, n)+H(n+1, \infty)\}\right), \\
K_{n-}=E_{r}\left(-(1 / 2) \tau_{s}(-n) \Psi ;-(1 / 2)\{H(-\infty,-n)+H(1-n, 0)\}\right), \\
\Psi=\sum_{j=1-r}^{0} \tau_{s}(j) \Phi \in \mathfrak{U}([1-r, r]), \\
d^{2 n} \varphi_{n}\left(Q^{\prime}\right)=p_{n}^{-1} \operatorname{tr}_{[1-n, n]}\left(Q^{\prime} \exp -\{H(1, n)+H(1-n, 0)\}\right), \\
d^{2 n} p_{n}=\operatorname{tr}_{[1-n, n]}(\exp -\{H(1, n)+H(1-n, 0)\}) .
\end{gathered}
$$

Proof. Since $K_{ \pm} \in \mathfrak{A}_{2}$ by (5.2) (iii), $K^{*} Q K \in \mathfrak{U}_{1}$ or $\mathfrak{U}_{2}$ according as $Q \in \mathfrak{U}_{1}$ or $\mathfrak{U}_{2}$. Since $\|\mathscr{L}(Q)\|_{n} \leqq\left\|K^{*} Q K\right\|_{n+1}, \mathscr{L}(Q) \in \mathfrak{A}_{1}$ or $\mathfrak{U}_{2}$. If $Q \geqq 0$, then $K^{*} Q K \geqq 0$ and hence $\varphi(\mathscr{L} Q)=\left(\tau_{c}(1)^{*} \varphi\right) \otimes\left(d^{-2} \operatorname{tr}_{[0,1]}\right)\left(K^{*} Q K\right) \geqq 0$, for any state $\varphi$ of $\mathfrak{U}$, where $\tau_{c}(1)^{*} \varphi$ is the state of $\mathfrak{U}(Z \backslash[0,1])$ such that $\tau_{c}(1)^{*} \varphi\left(Q^{\prime \prime}\right)=\varphi\left(\tau_{c}(1) Q^{\prime \prime}\right)$ for all $Q^{\prime \prime} \in \mathfrak{U}(Z \backslash[0,1])$. Hence $\mathscr{L} Q \geqq 0$. If 
$\mathscr{L} Q=0$ in addition, then $\varphi_{c}(\mathscr{L} Q)=\varphi_{c}\left(K^{*} Q K\right)=0$ for the central state $\varphi_{c}$ of $\mathfrak{A}$. Since $\varphi_{c}$ is faithful on the non negative elements of $\mathfrak{A}$, we have $K^{*} Q K=0$. Since $K_{ \pm}$has the inverses

$$
\begin{aligned}
& K_{+}^{-1}=E_{l}\left(\tau_{s}(1) \Phi / 2 ; H(2, \infty) / 2\right), \\
& K_{-}^{-1}=E_{l}\left(\tau_{s}(-r) \Phi / 2 ; H(-\infty,-1) / 2\right),
\end{aligned}
$$

we have $Q=0$. If $Q^{-1}$ exists, then $K^{*} Q K \geqq\left\|Q^{-1}\right\|^{-1}\left\|K^{-1}\right\|^{-2}$ and hence $\mathscr{L}(Q) \geqq\left\|Q^{-1}\right\|^{-1}\left\|K^{-1}\right\|^{-2}$. Therefore $\mathscr{L}(Q)^{-1} \in \mathfrak{A}$.

To obtain (iii), we use (5.10):

$$
\begin{aligned}
\prod_{j}^{1 \rightarrow N} \tau_{s}(j-1) K_{+} & =E_{r}[-(1 / 2) H(1, N+r) ;-(1 / 2) H(N+1, \infty)] \\
& =K_{n+} E_{r}[-(1 / 2) H(1, N) ;-(1 / 2) H(N+1, \infty)] .
\end{aligned}
$$

Since $H(1, N)$ commutes with every $\tau_{s}(j) \Phi$ in $H(N+1, \infty)$, the second factor of $(6.14)$ is $\exp -(1 / 2) H(1, N)$. Similar equation holds for $K_{-}$. Therefore

$$
\begin{gathered}
\mathscr{L}^{n} Q=\tau_{c}(n) d^{-2 n} \operatorname{tr}_{[1-n, n]}\left\{\left(K_{n+}^{\prime} K_{n-}^{\prime}\right)^{*} Q\left(K_{n+}^{\prime} K_{n-}^{\prime}\right)\right\}, \\
K_{n+}^{\prime}=\prod_{j}^{1 \rightarrow N} \tau_{s}(j-1) K_{+}, \\
K_{n-}^{\prime}=\prod_{j}^{1 \rightarrow N} \tau_{s}(1-j) K_{-} .
\end{gathered}
$$

This proves (iii).

Lemma 6.4. (i) Let $Q \in \mathfrak{A}, Q>0, \alpha(Q)<\infty$. Then

$$
\begin{gathered}
\alpha\left(\mathscr{L}^{n} Q\right) \leqq \alpha(Q) b(\|\Phi\|), \\
\alpha_{l}\left(\mathscr{L}^{n} Q\right) \leqq \alpha(Q) b^{\prime}(\|\Phi\|) \delta_{l-r}(\|\Phi\| / 2)+b(\|\Phi\|) \alpha_{l+n}(Q),
\end{gathered}
$$

where $\delta_{l}(\|\Phi\| / 2)$ is defined in (5.17) and $b^{\prime}(\|\Phi\|)$ is another constant. (ii) Let $\|Q\|_{M, x}\left\|Q^{-1}\right\| \leqq a, 0<a, x>1$. Then there exists $N(a, M, x,\|\Phi\|)$ such that

$$
\alpha\left(\mathscr{L}^{n} Q\right) \leqq 3 b(\|\Phi\|)
$$

for any $n \geqq N(a, M, x,\|\Phi\|)$ uniformly in $Q$.

Proof. From (5.12) and estimates in Theorem 4.2 (i), we have

$$
\begin{gathered}
\|S\| \leqq \exp \left\{F_{2 r-1}(\|\Phi\|)\|\Psi\| / 2\right\} \leqq \exp \left\{(r / 2) F_{2 r-1}(\|\Phi\|)\|\Phi\|\right\} \\
\left\|S^{-1}\right\| \leqq \exp \left\{(r / 2) F_{2 r-1}(\|\Phi\|)\|\Phi\|\right\}
\end{gathered}
$$


for $S=K_{n+}, K_{n-}, K_{n+}^{N N^{\prime}}, K_{n-}^{N N^{\prime}}$ where $r \leqq N<n, N^{\prime} \geqq r, N^{\prime}$ may become $+\infty$ and

$$
\begin{aligned}
K_{n+}^{N N^{\prime}}=E_{r}( & -\tau_{s}(n) \Psi / 2 ; \\
& \left.-\left\{H([n-N, n])+H\left(\left[n+1, n+1+N^{\prime}\right]\right)\right\} / 2\right), \\
K_{n-}^{N N^{\prime}}=E_{r}( & -\tau_{s}(-n) \Psi / 2 ; \\
& \left.-\left\{H\left(\left[-n-N^{\prime},-n\right]\right)+H([1-n, 1-n+N])\right\} / 2\right) .
\end{aligned}
$$

Hence

$\alpha\left(K_{n}^{*} Q K_{n}\right) \leqq \alpha(Q)\left\|\left(K_{n}^{*}\right)^{-1}\right\|\left\|K_{n}^{-1}\right\|\left\|K_{n}\right\|\left\|K_{n}^{*}\right\| \leqq \alpha(Q) b(\|\Phi\|)$,

where

$$
b(\|\Phi\|)=\exp \left\{4 r F_{2 r-1}(\|\Phi\|)\|\Phi\|\right\} .
$$

Since $b_{2} \geqq Q^{\prime \prime} \geqq b_{1}$ implies $b_{2} \geqq \varphi_{n}\left(Q^{\prime \prime}\right) \geqq b_{1}$, and since $\alpha\left(p_{n} \tau_{c}(n) Q^{\prime \prime \prime}\right)$ $=\alpha\left(Q^{\prime \prime \prime}\right)$, we have (6.18) for $n>r$.

If $n \leqq r, H(1, n)$ in (6.7) and $H(1-n, 0)$ in (6.8) are absent and $\Psi$ is replaced by $\Psi_{n} \equiv \sum_{j=1-n}^{0} \tau_{s}(j) \Phi$, which satisfies $\left\|\Psi_{n}\right\| \leqq r\|\Phi\|$. Hence we have the same result.

Since $\alpha(R) \geqq \alpha_{l}(R)$ for any $R$ by (3.16), the Eq. (6.19) for $l \leqq r$ follows from (6.18). The modification for the case $n \leqq r$ is the same as above. Hence we consider the case $n>r, l>r$.

Now we prove (6.19). Let $Q_{(n+l)}$ be such that

$$
Q_{(n+l)} \in \mathfrak{U}([-n-l, n+l]),\left\|Q-Q_{(n+l)}\right\|\left\|Q_{(n+l)}^{-1}\right\|=\alpha_{n+l}(Q) .
$$

Let

Let

$$
K_{(n, l)}=K_{n+}^{n-1, l-1} K_{n-}^{n-1, l-1}
$$

$$
Q_{l}^{\prime}=\tau_{c}(n) \varphi_{n}\left(K_{(n, l)}^{*} Q_{(n+l)} K_{(n, l)}\right) \in \mathfrak{U}([-l, l])
$$

and compute $\left\|Q^{\prime}-Q_{l}^{\prime}\right\|\left\|\left(Q_{l}^{\prime}\right)^{-1}\right\|$ where $Q^{\prime}=\tau_{c}(n) \varphi_{n}\left(K_{n}^{*} Q K_{n}\right)$. From Lemma 5.2 (i) and (6.21), we have

$$
\begin{aligned}
& \left\|K_{n}^{*} Q K_{n}-K_{(n, l)}^{*} Q K_{(n, l)}\right\| \\
& \leqq 4\|Q\| b(\|\Phi\|)^{1 / 2}\left\{(r / 2)\|\Phi\| F_{2 r-1}(\|\Phi\|)\right\} \delta_{l-r}(\|\Phi\| / 2) \\
& \quad\left\|K_{(n, l)}^{*}\left(Q-Q_{(n+l)}\right) K_{(n, l)}\right\| \leqq b(\|\Phi\|)^{1 / 2}\left\|Q-Q_{(n+l)}\right\| .
\end{aligned}
$$

Hence we have

$$
\begin{aligned}
\left\|Q^{\prime}-Q_{l}^{\prime}\right\| \leqq & \left(2 b(\|\Phi\|)^{1 / 2}\right)^{-1} b^{\prime}(\|\Phi\|) \delta_{l-r}(\|\Phi\| / 2) \\
& +b(\|\Phi\|)^{1 / 2}\left\|Q-Q_{(n+l)}\right\|,
\end{aligned}
$$


where

$$
b^{\prime}(\|\Phi\|)=b(\|\Phi\|)\left\{4 r\|\Phi\| F_{2 r-1}(\|\Phi\|)\right\} .
$$

On the other hand,

$$
\begin{gathered}
K_{(n, l)}^{*} Q_{(n+l)} K_{(n, l)} \geqq\left\|\left\{K_{(n, l)}^{*} Q_{(n+l)} K_{(n, l)}\right\}^{-1}\right\|^{-1} \\
\geqq b(\|\Phi\|)^{-1 / 2}\left\|Q_{(n+l)}^{-1}\right\|^{-1} .
\end{gathered}
$$

By Lemma 3.9 (3) and (1), we may assume

$$
\left\|Q_{(n+l)}^{-1}\right\| \leqq\left\|Q^{-1}\right\|\left(1+\alpha_{l}(Q)\right) \leqq 2\left\|Q^{-1}\right\| .
$$

Hence

$$
Q_{l}^{\prime} \geqq b(\|\Phi\|)^{-1 / 2}\left\|Q_{(n+l)}^{-1}\right\|^{-1} \geqq 2^{-1} b(\|\Phi\|)^{-1 / 2}\left\|Q^{-1}\right\|^{-1} .
$$

Therefore

$$
\begin{aligned}
\alpha_{l}\left(Q^{\prime}\right) & \leqq\left\|Q^{\prime}-Q_{l}^{\prime}\right\|\left\|Q_{l}^{\prime-1}\right\| \\
& \leqq b(\|\Phi\|) \alpha_{n+l}(Q)+b^{\prime}(\|\Phi\|) \alpha(Q) \delta_{l-r}(\|\Phi\| / 2) .
\end{aligned}
$$

Since $\alpha_{l}\left(p_{n} Q^{\prime}\right)=\alpha_{l}\left(Q^{\prime}\right)$, we have (6.19).

We now prove (ii). In the previous computation, we consider $Q_{(k)}$ instead of $Q_{(n+l)}$ and $K_{n}^{\prime}=K_{n+}^{N, \infty} K_{n-}^{N, \infty}$ instead of $K_{(n, l)}$. We then have bound (6.29) and (6.31) for $K_{n}^{*} Q K_{n}-\left(K_{n}^{\prime}\right)^{*} Q_{(k)} K_{n}^{\prime}$ and $\left(K_{n}^{\prime}\right)^{*} Q_{(k)} K_{n}^{\prime}$ where $l-r$ is to be replaced by $N-r+1$ and $(n+l)$ by $(k)$. Hence for any state $\varphi^{\prime}$ and $\varphi^{\prime \prime}$ of $\mathfrak{A}$, we have

$$
\begin{gathered}
\varphi^{\prime}\left(\tau_{c}(n) \varphi_{n}\left(K_{n}^{*} Q K_{n}\right)\right) \leqq \varphi^{\prime}\left[\tau_{c}(n) \varphi_{n}\left(\left(K_{n}^{\prime}\right)^{*} Q_{(k)} K_{n}^{\prime}\right)\right](1+\Delta) . \\
\varphi^{\prime \prime}\left(\tau_{c}(n) \varphi_{n}\left(K_{n}^{*} Q K_{n}\right)\right) \geqq \varphi^{\prime \prime}\left[\tau_{c}(n) \varphi_{n}\left(\left(K_{n}^{\prime}\right)^{*} Q_{(k)} K_{n}^{\prime}\right)\right](1-\Delta), \\
\Delta \leqq b(\|\Phi\|) \alpha_{k}(Q)+b^{\prime}(\|\Phi\|) \alpha(Q) \delta_{N-r+1}(\|\Phi\| / 2) .
\end{gathered}
$$

From $\|Q\|_{M, x}\left\|Q^{-1}\right\| \leqq a$, we have $\left\|Q^{-1}\right\|\|Q\|_{k} \leqq x^{-k} a$ for $k \geqq M$. By Lemma 3.9 (6), we have

$$
\alpha_{k}(Q) \leqq x^{-k} a\left(1-x^{-k} a\right)^{-1} .
$$

Let $L$ be an integer such that $L \geqq M$,

$$
x^{L} \geqq(1+4 b(\|\Phi\|)) a .
$$

For $k \geqq L$, we have $\alpha_{k}(Q) \leqq\{4 b(\|\Phi\|)\}^{-1}$. Since $\|Q\| \leqq\|Q\|_{M, x}$ we have $\alpha(Q) \leqq a$. Let $N$ be an integer such that $N>r$ and

$$
4 b^{\prime}(\|\Phi\|) a \delta_{N-r+1}(\|\Phi\| / 2) \leqq 1 .
$$


We then have $\Delta \leqq 1 / 2$ and hence by (6.5) and (3.13) we have $\alpha\left(\mathscr{L}^{n} Q\right) \leqq 3 \sup _{\varphi^{\prime} \varphi^{\prime \prime}} \varphi^{\prime}\left[\tau_{c}(n) \varphi_{n}\left(\left(K_{n}^{\prime}\right)^{*} Q_{(k)} K_{n}^{\prime}\right)\right] / \varphi^{\prime \prime}\left[\tau_{c}(n) \varphi_{n}\left(\left(K_{n}^{\prime}\right)^{*} Q_{(k)} K_{n}^{\prime}\right)\right]$.

Now we set $N(a, M, x,\|\Phi\|)=L+N+2$. For $n \geqq N(a, M, x,\|\Phi\|)$, we have $Q_{k} \in \mathfrak{U}([-k, k]), K_{n}^{\prime} \in \mathfrak{U}(Z \backslash[-k, k])$. Let

$$
\bar{\varphi}_{1}\left(Q^{\prime \prime}\right) \equiv \varphi_{1}\left[\tau_{c}(n) \varphi_{n}\left(Q_{(k)} Q^{\prime \prime}\right)\right]=\left(\varphi_{n} \otimes \tau_{c}(n)^{*} \varphi_{1}\right)\left(Q_{(k)} Q^{\prime \prime}\right)
$$

be a state on $\mathfrak{A}(Z \backslash[-k, k])\left(\ni Q^{\prime \prime}\right)$ induced by $\varphi_{1}=\varphi^{\prime}$ and $\varphi^{\prime \prime}$. Then we have

$$
\alpha\left(\mathscr{L}^{n} Q\right) \leqq 3 \sup _{\varphi^{\prime} \varphi^{\prime \prime}} \bar{\varphi}^{\prime}\left(\left(K_{n}^{\prime}\right)^{*} K_{n}^{\prime}\right) / \bar{\varphi}^{\prime \prime}\left(\left(K_{n}^{\prime}\right)^{*} K_{n}^{\prime}\right) \leqq 3 b(\|\Phi\|) .
$$

Lemma 6.5. $\mathscr{L}$ maps $\mathfrak{A}(M, x)$ continuously into itself where $x>1$.

Proof. By Lemma 6.3 (i), $\mathscr{L} 1 \in \mathfrak{A}_{1} \subset \mathfrak{U}(M, x)$. Now consider $Q$ such that $\|Q\|_{M, x} \leqq 1, Q=Q^{*}$. Let $Q^{\prime}=2+Q$. Then $3 \geqq Q^{\prime} \geqq 1$ and hence $\alpha\left(Q^{\prime}\right) \leqq 3$. By $(6.19)$,

$$
\alpha_{l}\left(\mathscr{L} Q^{\prime}\right) \leqq b(\|\Phi\|) \alpha_{l+1}\left(Q^{\prime}\right)+3 b^{\prime}(\|\Phi\|) \delta_{l-r}(\|\Phi\| / 2) .
$$

By $\left\|Q^{\prime}\right\|_{M, x} \leqq 1$, we have $\left\|Q^{\prime}\right\|_{n} \leqq x^{-n}$. By (3.21) we have

$$
\alpha_{l+1}\left(Q^{\prime}\right) \leqq x^{-(l+1)}\left(1-x^{-(l+1)}\right),
$$

where we have used $\left\|\left(Q^{\prime}\right)^{-1}\right\| \leqq 1$. Let $L$ be such that $y_{L}<1$ where

$$
y_{l}=b(\|\Phi\|) x^{-(l+1)}\left(1-x^{-(l+1)}\right)+3 b^{\prime}(\|\Phi\|) \delta_{l-r}(\|\Phi\| / 2) .
$$

From (6.1), we have

$$
\left\|\mathscr{L} Q^{\prime}\right\| \leqq\|K\|^{2}\left\|Q^{\prime}\right\| \leqq 3 b(\|\Phi\|)^{1 / 2} .
$$

By (3.22), $\|R\| \geqq\left\|R^{-1}\right\|^{-1}$ and (6.46),

$$
\left\|\mathscr{L} Q^{\prime}\right\|_{l} \leqq 3 b(\|\Phi\|)^{1 / 2} y_{l}\left(1-y_{l}\right)^{-1} .
$$

Thus

$$
\left\|\mathscr{L} Q^{\prime}\right\|_{M, x} \leqq 3 b(\|\Phi\|)^{1 / 2}\left\{1+|L-M|+\sum_{l=L}^{\infty} x^{l} y_{L}\left(1-y_{L}\right)^{-1}\right\}<\infty .
$$

Therefore $\|\mathscr{L} Q\|_{M, x} \leqq\left\|\mathscr{L} Q^{\prime}\right\|_{M, x}+2\|\mathscr{L} 1\|_{M, x}$ is uniformly bounded.

Let $Q=Q_{1}+i Q_{2}, Q_{1}^{*}=Q_{1}, Q_{2}^{*}=Q_{2}$. Then

$$
\left\|Q_{1}\right\|=\sup _{\varphi}\left|\varphi\left(Q_{1}\right)\right| \leqq \sup _{\varphi}\left|\varphi\left(Q_{1}\right)+i \varphi\left(Q_{2}\right)\right| \leqq\|Q\| .
$$

Similarly $\left\|Q_{2}\right\| \leqq\|Q\|$. Further, let $Q^{(l)}$ be such that $\left\|Q-Q^{(l)}\right\|=\|Q\|_{l}$, $Q^{(l)}=Q_{1}^{(l)}+i Q_{2}^{(l)},\left(Q_{1}^{(l)}\right)^{*}=Q_{1}^{(l)},\left(Q_{2}^{(l)}\right)^{*}=Q_{2}^{(l)}, Q_{1}^{(l)} \in \mathfrak{U}([-l, l]), Q_{2}^{(l)} \in \mathfrak{U}([-l, l])$. 
Then by the same argument as (6.49), we have

$$
\left\|Q_{1}\right\|_{l} \leqq\left\|Q-Q_{1}^{(l)}\right\| \leqq\left\|Q-Q^{(l)}\right\|=\|Q\|_{l} .
$$

Similarly $\left\|Q_{2}\right\|_{l} \leqq\|Q\|_{l}$. Therefore $\left\|Q_{1}\right\|_{M, x} \leqq\|Q\|_{M, x},\left\|Q_{2}\right\|_{M, x} \leqq\|Q\|_{M, x}$. By using the uniform boundedness for selfadjoint $Q$, we have the uniform boundedness of $\|\mathscr{L} Q\|_{M, x} \leqq\left\|\mathscr{L} Q_{1}\right\|_{M, x}+\left\|\mathscr{L} Q_{2}\right\|_{M, x}$.

\section{$\S$ 7. Convergence Proof}

Lemma 7.1. There exists a state $v$ of $\mathfrak{A}$ and $\lambda>0$ such that $v(\mathscr{L} Q)$ $=\lambda v(Q)$ for all $Q \in \mathfrak{A}$.

Proof. Let $\hat{\mathscr{L}}$ be the mapping of states of $\mathfrak{A}$ into themselves defined by

$$
(\hat{\mathscr{L}} \varphi)(Q)=\varphi(\mathscr{L} 1)^{-1} \varphi(\mathscr{L} Q) .
$$

Since

$$
\varphi(\mathscr{L} 1) \geqq\left\|(\mathscr{L} 1)^{-1}\right\|^{-1} \geqq b(\|\Phi\|)^{-1 / 2}>0,
$$

$\hat{\mathscr{L}}$ is weakly continuous. The set of states is convex and weakly compact. Hence $\hat{\mathscr{L}}$ has a fixed point $v$ due to the Schauder-Tychonov theorem. It satisfies

$$
v(\mathscr{L} Q)=\lambda v(Q), \quad \lambda=v(\mathscr{L} 1)>0 .
$$

Definition 7.2. Let $Q \in \mathfrak{A}, L Q=\lambda^{-1} \mathscr{L} Q . \Sigma(Q)$ denotes the closure of the convex hull of $\left\{L^{n} Q ; n=0,1,2, \ldots\right\}$.

Lemma 7.3. Let $Q>0, \alpha(Q)<\infty . \Sigma(Q)$ is a compact subset of $\mathfrak{U}$, convex and invariant under $L$. $L$ is continuous on $\mathfrak{A}$. If $Q \in \mathfrak{A}_{2}$, then $\Sigma(Q) \subset \mathfrak{A}_{2}$.

Proof. $\Sigma(Q)$ is convex because it is the closure of a convex set. Since $\|L(Q)\| \leqq \lambda^{-2}\|K\|^{2}\|Q\|, L$ is continuous. Since the convex hull of $\left\{L^{n} Q ; n=0,1,2, \ldots\right\}$ is invariant under $L$, its closure is also invariant due to the boundedness of $L$. We now show that $\Sigma(Q)$ is compact.

From (6.18) and (6.19), we have

$$
\begin{aligned}
\alpha\left(L^{n} Q\right) & =\alpha\left(\mathscr{L}^{n} Q\right) \leqq b(\|\Phi\|) \alpha(Q), \\
\alpha_{l}\left(L^{n} Q\right) & =\alpha_{l}\left(\mathscr{L}^{n} Q\right) \leqq b_{l}, \\
b_{l} & =\alpha(Q) b^{\prime}(\|\Phi\|) \delta_{l-r}(\|\Phi\| / 2)+b(\|\Phi\|) \alpha_{l}(Q),
\end{aligned}
$$

where we have used $\alpha_{l+n}(Q) \leqq \alpha_{l}(Q)$ (Eq. (3.17)). Since $\lim _{k \rightarrow \infty} \alpha_{k}(Q)=0$ for any $Q \in \mathfrak{A}, \lim _{l \rightarrow \infty} b_{l}=0$. Further $v\left(L^{n} Q\right)=v(Q)$. Therefore $\Sigma(Q)$ is compact due to Corollary 3.12 . 
Now assume $Q \in \mathfrak{A}_{2}$. Then $\sup l^{-1}\left\{\log \alpha_{l}(Q)+(l / r) \log l\right\}<+\infty$ and hence

$$
\begin{gathered}
\varlimsup_{l \rightarrow \infty} l^{-1}\left\{\log b_{l}+(l / r) \log l\right\}=\varlimsup_{l \rightarrow \infty} l^{-1}\left\{\log \max \left(\delta_{l-1}(\|\Phi\| / 2), \alpha_{l}(Q)\right)\right. \\
+(l / r) \log l\}<\infty
\end{gathered}
$$

From (3.22) and $\left\|R^{-1}\right\|^{-1} \leqq\|R\|$, we have

$$
\left\|L^{n} Q\right\|_{l} \leqq\left\|L^{n} Q\right\| b_{l}\left(1-b_{l}\right)^{-1},
$$

where $v\left(L^{n} Q\right)=v(Q)$ and (7.4) implies

$$
\left\|L^{n} Q\right\| \leqq v(Q) \alpha(Q) b(\|\Phi\|) .
$$

(7.7) and (7.8) give a uniform bound for $L^{n} Q$, which is preserved in taking convex hull and closure. Thus $Q^{\prime} \in \Sigma(Q)$ satisfies

$$
\left\|Q^{\prime}\right\|_{l} \leqq v(Q) \alpha(Q) b(\|\Phi\|) b_{l}\left(1-b_{l}\right)^{-1} .
$$

By (7.6), we see that $Q^{\prime} \in \mathfrak{A}_{2}$. Q.E.D.

Remark 7.4. $\Sigma(Q)$ is compact for any $Q$. This is because

$$
Q=Q_{1}-Q_{2}+i\left(Q_{3}-Q_{4}\right)
$$

where $Q_{1}=\left(Q+Q^{*}\right) / 2+2\|Q\| \geqq\|Q\|, Q_{3}=i\left(Q^{*}-Q\right) / 2+2\|Q\| \geqq\|Q\|$ and $Q_{2}=Q_{4}=2\|Q\|$. The estimates like (7.7) and (7.8) hold for each $Q_{j}$ and hence $\Sigma(Q)$ is compact by Lemma 3.2.

Lemma 7.5. There exists $h \in \mathfrak{A}_{2}$ such that

$$
L h=h, v(h)=1, \alpha(h) \leqq b(\|\Phi\|) .
$$

Proof. $\Sigma(1)$ has a fixed point $h$ under the mapping $L$ by Lemma 7.3 and the Schauder-Tychonov theorem, and $h \in \mathfrak{A}_{2}$. Since $\alpha(1)=1,(7.4)$ implies $\alpha(h) \leqq b(\|\Phi\|)$. Q.E.D.

Lemma 7.6. Let

$$
E Q=v(Q) h
$$

Then

$$
\lim _{n \rightarrow \infty}\left(\varrho_{x}\right)^{n}\left\|L^{n}(1-E)\right\|_{1, x}=0
$$

for any $x>1$ and some $\varrho_{x}>1$.

Proof. Note that $E^{2}=E$. Since $L$ and $E$ are linear operators on $\mathfrak{A}_{M, x}$, it is enough to prove the convergence of

$$
\lim _{n \rightarrow \infty} \varrho_{x}^{n}\left\|L^{n} Q\right\|_{1, x}=0
$$

uniformly in $Q$ such that $\|Q\|_{1, x} \leqq 1$ and $(1-E) Q=Q$. The latter condition is the same as $v(Q)=0$. Any $Q$ can be decomposed as $Q=Q_{1}$ 
$+i Q_{2}, Q_{1}^{*}=Q_{1}, Q_{2}^{*}=Q_{2}$ and by the proof of Lemma 6.5, we have $\left\|Q_{1}\right\|_{M, x} \leqq\|Q\|_{M, x}=1,\left\|Q_{2}\right\|_{M, x} \leqq\|Q\|_{M, x}=1$. Further $v(Q)=v\left(Q_{1}\right)$ $+i v\left(Q_{2}\right)=0$ implies $v\left(Q_{1}\right)=v\left(Q_{2}\right)=0$. Therefore it suffices to prove the uniform convergence of (7.14) for $Q$ such that $Q^{*}=Q, v(Q)=0,\|Q\|_{1, x} \leqq 1$. We already know by Lemma 6.5 that $L$ is a continuous map of $\mathfrak{A}_{M, x}$ into $\mathfrak{U}_{M, x}$. Let $Q^{\prime}=Q+2, \Lambda_{0}\left(Q^{\prime}\right)=Q^{\prime}$ and

$$
\Lambda_{n}\left(Q^{\prime}\right)=L^{N} \Lambda_{n-1}\left(Q^{\prime}\right)-[6 b(\|\Phi\|)]^{-1} v\left(\Lambda_{n-1}\left(Q^{\prime}\right)\right),
$$

$n=1,2, \ldots$. We fix an $N$ such that

$$
N \geqq N(a, M, x,\|\Phi\|), \quad N \geqq M,
$$

where $N(a, M, x,\|\Phi\|)$ is given in Lemma 6.4 (ii),

$$
a=2(6 b(\|\Phi\|)-1) \text {, }
$$

and $M$ is chosen so as to satisfy

$$
\begin{gathered}
x^{M} \geqq 2 a, \\
\sum_{l=M}^{\infty} x^{l} b^{\prime}(\|\Phi\|) \delta_{l-r}(\|\Phi\| / 2) \leqq 1 / 10 .
\end{gathered}
$$

We now prove the following properties of $\Lambda_{n}\left(Q^{\prime}\right)$ and $\Lambda_{n}(2)$.

$$
\begin{gathered}
\Lambda_{n}\left(Q^{\prime}\right) \geqq 0, \\
\Lambda_{n}(2) \geqq 0, \\
\Lambda_{n}\left(Q^{\prime}\right)-\Lambda_{n}(2)=L^{n N} Q, \\
v\left(\Lambda_{n}\left(Q^{\prime}\right)\right)=2 \bar{\varrho}_{x}^{-n N}=v\left(\Lambda_{n}(2)\right), \\
\bar{\varrho}_{x}=\left[1-\{6 b(\|\Phi\|)\}^{-1}\right]^{-1 / N} . \\
\left\|\Lambda_{n}\left(Q^{\prime}\right)\right\|_{M, x}\left\|\Lambda_{n}\left(Q^{\prime}\right)^{-1}\right\| \leqq a, \\
\left\|\Lambda_{n}(2)\right\|_{M, x}\left\|\Lambda_{n}(2)^{-1}\right\| \leqq a .
\end{gathered}
$$

First consider $n=0$. Since $v\left(Q^{\prime}\right)=2=v(2)$ due to $v(Q)=0$, we have (7.23). Since $\|Q\| \leqq\|Q\|_{M, x} \leqq 1$, we have $1 \leqq Q^{\prime} \leqq 3$. Hence (7.20) (7.21) holds. (7.22) is obvious. Since $\left\|Q^{\prime}\right\|_{M, x} \leqq 2+\|Q\|_{M, x} \leqq 3 \leqq a$, we have (7.25). $\|2\|\left\|_{M, x}\right\| 2^{-1} \|=1 \leqq a$.

Next assume (7.20) (7.26) for $n=k-1$ and consider (7.20) (7.26) for $n=k$. By definition (7.15), (7.22) holds, where we use (7.23) for $n=k-1$. Further, from (7.15),

$$
v\left(\Lambda_{k}\left(Q^{\prime}\right)\right)=\left\{1-[12 b(\|\Phi\|)]^{-1}\right\} v\left(\Lambda_{k-1}\left(Q^{\prime}\right)\right)=2 \bar{\varrho}_{x}^{-k N}
$$

and the same holds when $Q^{\prime}$ is replaced by 2 . This proves (7.23) for $n=k$. 
From (7.16), (7.25) and Lemma 6.4 (ii), we have

$$
\alpha\left(L^{N} \Lambda_{k-1}\left(Q^{\prime}\right)\right) \leqq 3 b(\|\Phi\|) .
$$

Therefore

$$
\begin{aligned}
L^{N} \Lambda_{k-1}\left(Q^{\prime}\right) & \geqq[3 b(\|\Phi\|)]^{-1} v\left(L^{N} \Lambda_{k-1}\left(Q^{\prime}\right)\right) \\
& =[3 b(\|\Phi\|)]^{-1} v\left(\Lambda_{k-1}\left(Q^{\prime}\right)\right) .
\end{aligned}
$$

From (7.29) and (7.15), we have

$$
\Lambda_{k}\left(Q^{\prime}\right) \geqq v\left(\Lambda_{k-1}\left(Q^{\prime}\right)\right)[6 b(\|\Phi\|)]^{-1} .
$$

Then same holds when $Q^{\prime}$ is replaced by 2 . Hence we have (7.20) and (7.21) for $n=k$.

From (7.29), we have

$$
[6 b(\|\Phi\|)]^{-1} v\left(\Lambda_{k-1}\left(Q^{\prime}\right)\right)\left\|\left\{L^{N} \Lambda_{k-1}\left(Q^{\prime}\right)\right\}^{-1}\right\| \leqq 1 / 2 .
$$

Therefore, by (3.26) and (7.28), we have

$$
\alpha\left(\Lambda_{k}\left(Q^{\prime}\right)\right) \leqq 2 \alpha\left(L^{N} \Lambda_{k-1}\left(Q^{\prime}\right)\right)-1 \leqq 6 b(\|\Phi\|)-1 .
$$

From Lemma 6.4 (i),

$$
\begin{gathered}
\alpha_{l}\left(L^{N} \Lambda_{k-1}\left(Q^{\prime}\right)\right) \leqq b_{l}, \\
b_{l} \equiv \alpha\left(\Lambda_{k-1}\left(Q^{\prime}\right)\right) b^{\prime}(\|\Phi\|) \delta_{l-r}(\|\Phi\| / 2)+b(\|\Phi\|) \alpha_{l+N}\left(\Lambda_{k-1}\left(Q^{\prime}\right)\right) .
\end{gathered}
$$

From (7.18) and (7.25) with $n=k-1$, we have

$$
\left\|\Lambda_{k-1}\left(Q^{\prime}\right)\right\|_{l+N}\left\|\Lambda_{k-1}\left(Q^{\prime}\right)^{-1}\right\| \leqq x^{-M} a \leqq 1 / 2
$$

for $l \geqq M$. By (3.21) and (7.35),

$$
\alpha_{l+N}\left(\Lambda_{k-1}\left(Q^{\prime}\right)\right) \leqq 2\left\|\Lambda_{k-1}\left(Q^{\prime}\right)\right\|_{l+N}\left\|\Lambda_{k-1}\left(Q^{\prime}\right)^{-1}\right\| .
$$

Hence

$$
\sum_{l=M}^{\infty} x^{l} \alpha_{l+N}\left(\Lambda_{k-1}\left(Q^{\prime}\right)\right) \leqq 2 x^{-N}\left\|\Lambda_{k-1}\left(Q^{\prime}\right)\right\|_{M+N, x}\left\|\Lambda_{k-1}\left(Q^{\prime}\right)^{-1}\right\| .
$$

From (7.18), we have $2 b(\|\Phi\|) x^{-M} \leqq a^{-1} b(\|\Phi\|) \leqq 10^{-1}$. Therefore, by (7.19), (7.34), (7.37) and (7.25), we have

$$
\sum_{l=M}^{\infty} x^{l} b_{l} \leqq a / 10 .
$$

From (7.38), it follows for $l \geqq M$

$$
b_{l} \leqq x^{-M} a / 10 \leqq 20^{-1} .
$$


From (3.25), (7.31) and (7.39),

$$
\alpha_{l}\left(\Lambda_{k}\left(Q^{\prime}\right)\right) \leqq b_{l}\left\{1-\left(1+b_{l}\right) / 2\right\}^{-1} \leqq(40 / 19) b_{l} .
$$

From (7.40), (7.39) and (3.22), we have

$\left\|\Lambda_{k}\left(Q^{\prime}\right)\right\|_{l}\left\|\Lambda_{k}\left(Q^{\prime}\right)^{-1}\right\| \leqq(40 / 19) b_{l}\left(1-(40 / 19) b_{l}\right)^{-1} \leqq(40 / 17) b_{l}$.

By (7.38), (7.41), and (7.32), we have

$$
\left\|\Lambda_{k}\left(Q^{\prime}\right)\right\|_{M, x}\left\|\Lambda_{k}\left(Q^{\prime}\right)^{-1}\right\| \leqq a .
$$

This proves (7.25) for $n=k$. The same calculation with $Q^{\prime}$ replaced by 2 yields (7.26). This completes the inductive proof of $(7.20) \sim(7.26)$.

From (7.25) and (7.28), we have

$$
\left\|\Lambda_{n}\left(Q^{\prime}\right)\right\|_{M, x} \leqq a\left\|\Lambda_{n}\left(Q^{\prime}\right)^{-1}\right\| \leqq a v\left(\Lambda_{n}\left(Q^{\prime}\right)\right) \leqq 2 a \bar{\varrho}_{x}^{-n N} .
$$

Similarly,

$$
\left\|\Lambda_{n}(2)\right\|_{M, x} \leqq 2 a \bar{\varrho}_{x}^{-n N} .
$$

From (7.22), (7.43) and (7.44), we have

$$
\left\|L^{n N} Q\right\|_{M, x} \leqq 4 a \bar{\varrho}_{x}^{-n N} .
$$

Hence

$$
\lim _{n \rightarrow \infty}\left\|L^{n N} Q\right\|_{M, x} \varrho_{x}^{n N}=0
$$

for any $\varrho_{x}<\bar{\varrho}_{x}$. This then implies, due to the boundedness of each $L$ (Lemma 6.5),

$$
\lim _{n \rightarrow \infty}\left\|L^{n N+k} Q\right\|_{M, x} \varrho_{x}^{n N+k}=0
$$

for $k=0,1, \ldots, N-1$. Hence we have

$$
\lim _{n \rightarrow \infty}\left\|L^{n} Q\right\|_{M, x} \varrho_{x}^{n}=0 .
$$

Since $\|Q\|_{l} \leqq\|Q\| \leqq\|Q\|_{M, x}$ for $l=1, \ldots, M-1$, we have

$$
\|Q\|_{1, x} \leqq M\|Q\|_{M, x} .
$$

Therefore we have (7.13). Q.E.D.

\section{§ 8. Gibbs States}

Lemma 8.1. Let $Q \in \mathfrak{A}_{1, x}$ for some $x$. Let $\varphi_{c}$ be the central state of $\mathfrak{A}$. Let

$$
\begin{gathered}
\bar{\varphi}_{n}^{n_{1}, n_{2}}(Q)=\varphi_{c}\left(Q \exp \left\{\Delta_{1}^{-}+\Delta_{0}^{+}-U\left(1, n+n_{1}\right)-U\left(1-n-n_{2}, 0\right)\right\}\right) \bar{Z}_{n}^{-1}, \\
\bar{Z}_{n}=\varphi_{c}\left(\exp \left\{\Delta_{1}^{-}+\Delta_{0}^{+}-U\left(1, n+n_{1}\right)-U\left(1-n-n_{2}, 0\right)\right\}\right) .
\end{gathered}
$$


Then

$$
\begin{gathered}
\lim _{n \rightarrow \infty} \bar{\varphi}_{n}^{n_{1}, n_{2}}(Q)=v(Q), \\
\lim _{n \rightarrow \infty} \frac{1}{2 n} \log \bar{Z}_{n}=\frac{1}{2} \log \lambda .
\end{gathered}
$$

Proof. By (5.8), we have

$$
\begin{gathered}
\exp (1 / 2)\left\{\Delta_{1}^{-}+\Delta_{0}^{+}-U\left(1, n+n_{1}\right)-U\left(1-n-n_{2}, 0\right)\right\} \\
=K_{N}^{\prime} \exp -(1 / 2)\left\{H(1, N)+H(1-N, 0)+H^{\prime}\right\}, \\
H^{\prime}=H_{+}^{\prime}+H_{-}^{\prime}, \\
H_{+}^{\prime}=H\left(N+1, n+n_{1}\right)+\Delta_{n+n_{1}}^{+}, \\
H_{-}^{\prime}=H\left(1-n-n_{2},-N\right)+\Delta_{-n-n_{2}}^{-}, \\
K_{N}^{\prime}=K_{N+}^{\prime} K_{N-}^{\prime}, \\
K_{N+}^{\prime}=E_{r}\left(-\tau_{s}(N) \Psi / 2 ;-\left\{H(1, N)+H_{+}^{\prime}\right\} / 2\right), \\
K_{N-}^{\prime}=E_{r}\left(-\tau_{s}(-N) \Psi / 2 ;-\left\{H(1-N, 0)+H_{-}^{\prime}\right\} / 2\right),
\end{gathered}
$$

where $n>N+r$. Let

$$
\begin{gathered}
\bar{\varphi}_{n-N}^{\prime}\left(Q^{\prime}\right)=\varphi_{c}\left(Q^{\prime} \exp -\tau_{c}(N) H^{\prime}\right)\left(\bar{Z}_{n-N}^{\prime}\right)^{-1}, \\
\bar{Z}_{n-N}^{\prime}=\varphi_{c}\left(\exp -\tau_{c}(N) H^{\prime}\right) .
\end{gathered}
$$

We then have

$$
\bar{\varphi}_{n}^{n_{1} n_{2}}(Q)=\bar{Z}_{n}^{-1} \bar{Z}_{n-N}^{\prime} \bar{\varphi}_{n-N}^{\prime}\left[\tau_{c}(N) p_{N} \varphi_{N}\left(\left(K_{N}^{\prime}\right)^{*} Q K_{N}^{\prime}\right)\right]
$$

where $p_{n}$ and $\varphi_{n}$ are defined in Lemma 6.3 (iii).

We prove (8.3) for positive $Q$ such that $2 \geqq Q \geqq 1$. (8.3) for a general $Q$ will immediately follow from this case by linearity.

Let $B$ be a constant such that

$$
\left\|\left(K_{N}^{\prime}\right)\right\| \leqq B, \quad\left\|\left(K_{N}\right)\right\| \leqq B, \quad\left\|\left(K_{N}\right)^{-1}\right\| \leqq B
$$

Given $\varepsilon$, there exists $L_{1}(\varepsilon)$ such that for $n-N>L_{1}(\varepsilon)$

$$
\left\|K_{N}^{\prime}-K_{N}\right\|<\varepsilon .
$$

We then have

$$
\left\|\varphi_{N}\left(\left(K_{N}^{\prime}\right)^{*} Q K_{N}^{\prime}\right)-\varphi_{N}\left(K_{N}^{*} Q K_{N}\right)\right\|\left\|\varphi_{N}\left(K_{N}^{*} Q K_{N}\right)^{-1}\right\| \leqq 4 B^{3} \varepsilon .
$$

There also exists $L_{2}(\varepsilon)$ such that for $N>L_{2}(\varepsilon)$

$$
\left\|\lambda^{-N} \tau_{c}(N) p_{N} \varphi_{N}\left(K_{N}^{*} Q K_{N}\right)-v(Q) h\right\|<\varepsilon .
$$


Since $\left\|\left\{\lambda^{-N} \tau_{c}(N) p_{N} \varphi_{N}\left(K_{N}^{*} Q K_{N}\right)\right\}^{-1}\right\|^{-1} \leqq\left\|h^{-1}\right\|^{-1}+\varepsilon$ by $(8.18)$, we have $\left\|\lambda^{-N} \tau_{c}(N) p_{N} \varphi_{N}\left(\left(K_{N}^{\prime}\right)^{*} Q K_{N}^{\prime}\right)-v(Q) h\right\|\left\|h^{-1}\right\|$

$$
\leqq\left\{4 B^{3}\left(1+\varepsilon\left\|h^{-1}\right\|\right)+\left\|h^{-1}\right\|\right\} \varepsilon=\varepsilon_{1} .
$$

Similarly

$$
\begin{aligned}
&\left\|\lambda^{-N} \tau_{c}(N) p_{N} \varphi_{N}\left(\left(K_{N}^{\prime}\right)^{*} K_{N}^{\prime}\right)-h\right\|\left\|h^{-1}\right\| \\
& \leqq\left\{2 B^{3}\left(1+\varepsilon\left\|h^{-1}\right\|\right)+\left\|h^{-1}\right\|\right\} \varepsilon=\varepsilon_{2} .
\end{aligned}
$$

For any state $\varphi_{1}$, we have

$$
\begin{gathered}
\left|\varphi_{1}\left(\lambda^{-N} p_{N} \tau_{c}(N) \varphi_{N}\left(K_{N}^{\prime *} Q K_{N}^{\prime}\right)\right) /\left[v(Q) \varphi_{1}(h)\right]-1\right| \leqq \varepsilon_{1}, \\
\left|\varphi_{1}\left(\lambda^{-N} \mathrm{p}_{N} \tau_{c}(N) \varphi_{N}\left(K_{N}^{* *} K_{N}^{\prime}\right)\right) /\left[\varphi_{1}(h)\right]-1\right| \leqq \varepsilon_{2} .
\end{gathered}
$$

Therefore for $n \geqq L_{1}(\varepsilon)+L_{2}(\varepsilon)$,

$$
\left|\bar{\varphi}_{n}^{n_{1} n_{2}}(Q)\left[\bar{\varphi}_{n}^{n_{1} n_{2}}(1) v(Q)\right]^{-1}-1\right|<\left(\varepsilon_{1}+\varepsilon_{2}\right)\left(1-\varepsilon_{2}\right)^{-1} .
$$

Since $\bar{\varphi}_{n}^{n_{1} n_{2}}(1)=1$ for all $n$, we have

$$
\lim _{n \rightarrow \infty} \bar{\varphi}_{n}^{n_{1} n_{2}}(Q) / v(Q)=1 .
$$

We note that the convergence is uniform in $n_{1}$ and $n_{2}$.

Next we prove (8.4). In (8.14), we set $Q=1, n-N=L$. Given $\varepsilon$, we choose $L>L_{1}(\varepsilon)$, and for this $L$ we choose $L_{3}(\varepsilon)$ such that $L_{3}(\varepsilon)^{-1} L<\varepsilon$, $L_{3}(\varepsilon)^{-1}\left|\log \bar{Z}_{L}^{\prime}\right|<\varepsilon$. We then have, for $n-N=L$ and $N>\max \left(L_{3}(\varepsilon)\right.$, $\left.L_{2}(\varepsilon)\right)$,

$\left|n^{-1} \log \bar{Z}_{n}-\log \lambda\right|$

$\leqq n^{-1}\left|\log \bar{Z}_{L}^{\prime}\right|+n^{-1} L|\log \lambda|$

$+n^{-1}\left|\log \bar{\varphi}_{L}^{\prime}\left[\lambda^{-N} \tau_{c}(N) p_{N} \varphi_{N}\left(K_{N}^{*} K_{N}^{\prime}\right)\right]\right|$

$<(1+\log \lambda) \varepsilon+L_{3}(\varepsilon)^{-1}\left|\log \bar{\varphi}_{L}^{\prime}(h)\right|+L_{3}(\varepsilon)^{-1} \max \left\{\log \left(1+\varepsilon_{2}\right),-\log \left(1-\varepsilon_{2}\right)\right\}$.

Since $\left|\log \bar{\varphi}_{L}^{\prime}(h)\right| \leqq \max \left\{|\log \|h\||,\left|\log \left\|h^{-1}\right\|\right|\right\}$, we have (8.4). Q.E.D.

Lemma 8.2. Let $Q \in \mathfrak{U}_{0}$ and

$$
F=E_{r}(-\Psi / 2 ;-\{H(-\infty, 0)+H(1, \infty)\} / 2) \text {. }
$$

Then

$$
\begin{gathered}
\lim _{a \rightarrow-\infty, b \rightarrow \infty} \varphi_{a b}^{G}(Q)=v\left(F^{*} Q F\right) / v\left(F^{*} F\right), \\
\lim _{b \rightarrow a \rightarrow \infty}(b-a)^{-1} \log Z(a, b)=\frac{1}{2} \log \lambda+\log d
\end{gathered}
$$

where $\varphi_{a b}^{G}$ and $Z(a, b)$ are given by (2.2) and (2.3). 
Proof. Let $a<-r, b>r$ and

$$
F_{a b}=E_{r}\left(-\Psi / 2 ;\left\{\Delta_{0}^{+}+\Delta_{1}^{-}-U(a, 0)-U(1, b)\right\} / 2\right)
$$

Then

$$
\varphi_{a b}(Q)=\bar{\varphi}_{n}^{n_{1} n_{2}}\left(F_{a b}^{*} Q F_{a b}\right) / \bar{\varphi}_{n}^{n_{1} n_{2}}\left(F_{a b}^{*} F_{a b}\right)
$$

where $a=1-\left(n+n_{2}\right), b=n+n_{1}$. By a variance of Lemma 5.2,

$$
\lim _{n \rightarrow \infty}\left\|F_{a b}-F\right\|=0
$$

uniformly in $n_{1}$ and $n_{2}$. Furthermore $\left\|F^{-1}\right\|<\infty$ and hence $v\left(F^{*} F\right)^{-1}$ $\leqq\left\|F^{-1}\right\|^{2}<\infty$. By Lemma 8.1, we obtain (8.27).

To prove (8.28), we note the formula

$$
Z(a, b)=\bar{Z}_{n} \bar{\varphi}_{n}^{n_{1} n_{2}}\left(F_{a b}^{*} F_{a b}\right) d^{\left(2 n+n_{1}+n 2\right)},
$$

if $a=1-n-n_{2}, b=n+n_{1}$. Since $\bar{\varphi}_{n}^{n_{1} n_{2}}\left(F_{n}^{*} F_{n}\right)$ converges to a non zero constant $v\left(F^{*} F\right)$, we have

$$
\begin{aligned}
\lim _{n \rightarrow \infty} & \frac{1}{2 n} \log Z\left(-\left(n+n_{1}\right), n+n_{2}\right) \\
& =\lim _{n \rightarrow \infty} \frac{1}{2 n} \log \bar{Z}_{n}+\log d=\frac{1}{2} \log \lambda+\log d .
\end{aligned}
$$

Since $Z(a, b)$ depends only on $b-a$, we have (8.28). Q.E.D.

This lemma proves Theorem 2.1 except for the analyticity.

Lemma 8.3. $\varphi_{\Phi}(Q)$ is lattice translation invariant.

Proof. The following two quantities coincide for $n>0$.

$$
\begin{aligned}
v\left(F^{*} F\right) \varphi_{\Phi}(Q) & =\lim _{N \rightarrow \infty} \bar{\varphi}_{N}^{0, n}(Q), \\
v\left(F^{*} F\right) \varphi_{\Phi}\left(\tau_{s}(n) Q\right) & =\lim _{N \rightarrow \infty} \bar{\varphi}_{N}^{n, 0}\left(\tau_{s}(n) Q\right) .
\end{aligned}
$$

Hence $\varphi_{\Phi}(Q)=\varphi_{\Phi}\left(\tau_{s}(n) Q\right)$. Q.E.D.

Lemma 8.4. $\varphi_{\Phi}(Q)$ is time translation invariant.

Proof. We have

$$
\varphi_{a, b}^{G}([U(a, b), Q])=0 .
$$

Hence

$$
\varphi_{\Phi}([\delta(U(Z)) Q])=0 .
$$

Hence $\varphi_{\Phi}\left(\tau_{T}(t) Q\right)=0$. Q.E.D. 
Lemma 8.5. $\varphi_{\Phi}(Q)$ satisfies the $K M S$ condition.

Proof. Let $Q_{1} \in \mathfrak{U}_{0}, Q_{2} \in \mathfrak{U}_{0}$. We have

$$
\varphi_{a, b}^{G}\left(\left\{\exp \delta(U(a, b)) Q_{2}\right\} Q_{1}\right)=\varphi_{a, b}^{G}\left(Q_{1} Q_{2}\right) .
$$

Hence we have

$$
\varphi_{\Phi}\left(\left\{\exp \delta(U(Z)) Q_{2}\right\} Q_{1}\right)=\varphi_{\Phi}\left(Q_{1} Q_{2}\right) .
$$

By continuity, it holds for any $Q_{2} \in \mathfrak{A}_{1}$. Hence

By Lemma 4.2, $\exp \delta(-s U(Z)) \tau_{T}(t) Q_{2}$ is holomorphic in $t+i s$.

$$
\int \exp \delta(U(Z))\left(\tau_{T}(t) Q_{2}\right) f_{0}(t) \mathrm{dt}=\int \tau_{T}(t) Q_{2} f_{1}(t) \mathrm{dt}
$$

where $f_{\alpha}(t)=\int_{-\infty}^{\infty} e^{-i s t+\alpha s} f(s) \mathrm{ds}$ is holomorphic in $t+i \alpha$. Note that $\tau_{T}(t) Q_{2} \in \mathfrak{A}_{1}$. Hence we have $\varphi_{\Phi}\left(Q_{1} Q_{2}\left(f_{0}\right)\right)=\varphi_{\Phi}\left(Q_{2}\left(f_{1}\right) Q_{1}\right)$ for $Q_{1}, Q_{2}$ $\in \mathfrak{A}_{0}$. By continuity, this equation holds for any $Q_{1}, Q_{2} \in \mathfrak{U}$. Hence $\varphi_{\Phi}$ satisfies the $K M S$ condition.

Lemma 8.6. $\varphi_{\Phi}(Q)$ has a uniform exponential clustering property.

Proof. Let $Q_{1} \in \mathfrak{A}_{1}$. Given $\varepsilon>0$, we prove the existence of $N_{\varepsilon}$ such that for $N>N_{\varepsilon}$ and $Q_{2} \in \mathfrak{A}(Z \backslash[-N, N])$, we have

$$
\left|\varphi_{\Phi}\left(Q_{1} Q_{2}\right)-\varphi_{\Phi}\left(Q_{1}\right) \varphi_{\Phi}\left(Q_{2}\right)\right| e^{\varrho N}<\varepsilon\left\|Q_{2}\right\|
$$

where $\varrho$ is some positive constant.

We first prove the corresponding property for $v$. Let $Q \in \mathfrak{A}_{1}, v(Q)=0$. Let $x>1$ and $\varrho<\left(\log \varrho_{x}\right) / 2, \varrho<\log x, \varrho>0$.

Since $v\left(Q^{\prime}\right)=v\left(L^{n} Q^{\prime}\right)$ and $\alpha\left(L^{n} Q^{\prime}\right)=\alpha\left(\mathscr{L}^{n} Q^{\prime}\right) \leqq \alpha\left(Q^{\prime}\right) b(\|\Phi\|)$ by (6.18), we have $\left\|L^{n} Q^{\prime}\right\| \leqq \alpha\left(Q^{\prime}\right) b(\|\Phi\|) v\left(Q^{\prime}\right)$ if $Q^{\prime}>0$. If $Q^{\prime}$ is selfajoint and $\left\|Q^{\prime}\right\| \leqq 1$, then $\left\|2+Q^{\prime}\right\| \leqq 3, \alpha\left(2+Q^{\prime}\right) \leqq 3$. Hence $\left\|L^{n}\left(2+Q^{\prime}\right)\right\| \leqq 9 b(\|\Phi\|)$. Similarly $\left\|L^{n} 1\right\| \leqq b(\|\Phi\|)$. Hence $\left\|L^{n} Q^{\prime}\right\| \leqq 11 b(\|\Phi\|)$. For general $Q^{\prime}$, $Q^{\prime}=Q_{1}+i Q_{2}, Q_{1}^{*}=Q_{1}, Q_{2}^{*}=Q_{2},\left\|Q_{1}\right\| \leqq\left\|Q^{\prime}\right\|,\left\|Q_{2}\right\| \leqq\left\|Q^{\prime}\right\|$. Hence

$$
\left\|L^{n}\left(Q^{\prime}\right)\right\| \leqq 22 b(\|\Phi\|)\left\|Q^{\prime}\right\|
$$

for any $Q^{\prime} \in \mathfrak{A}$.

Let $K_{n}$ be given by (6.6), $K_{n \pm}^{N N^{\prime}}$ by (6.23), (6.24), $K_{(n, l)}=K_{n+}^{n-1, l-1} K_{n-}^{n-1, l-1}$. There exists $L_{1}(\varepsilon)$ such that for $l \geqq L_{1}(\varepsilon)$

$$
e^{2 \varrho l}\left\|K_{(n, l)} K_{n}^{-1}-1\right\| \leqq \varepsilon,
$$

due to $\left\|K_{(n, l)} K_{n}^{-1}-1\right\| \leqq\left\|K_{(n, l)}-K_{n}\right\|\left\|K_{n}^{-1}\right\|$ and Lemma 5.2. Since $Q \in \mathfrak{U}_{1}$, there exists $L_{2}(\varepsilon)$ such that for $l \geqq L_{2}(\varepsilon)$

$$
e^{\dot{e} l}\left\|Q-Q^{(l)}\right\|<\varepsilon, \quad Q^{(l)} \in \mathfrak{A}([-l, l]) .
$$


By Lemma 7.6, there exists $L_{3}(\varepsilon)$ such that for $l \geqq L_{3}(\varepsilon)$

$$
e^{2 \varrho l}\left\|L^{l} Q\right\| \leqq e^{2 \varrho l}\left\|L^{l} Q\right\|_{1, x}<\varepsilon
$$

due to $v(Q)=0$.

We now have the following series of estimates. Let $N \geqq L(\varepsilon)$ $=\max \left(L_{1}(\varepsilon), L_{2}(\varepsilon), L_{3}(\varepsilon)\right)$ and $Q_{2} \in \mathfrak{A}(Z \backslash[-2 N, 2 N])$. By (8.43) and (8.44),

$$
\begin{gathered}
e^{2 \varrho N}\left\|Q-\left(K_{N}^{*}\right)^{-1} K_{(N, N)}^{*} Q^{(2 N)} K_{(N, N)} K_{N}^{-1}\right\|<\varepsilon^{\prime}, \\
e^{2 \varrho N}\left\|Q Q_{2}-\left(K_{N}^{*}\right)^{-1} K_{(N, N)}^{*} Q^{(2 N)} Q_{2} K_{(N, N)} K_{N}^{-1}\right\|<\varepsilon^{\prime}\|Q\|, \\
\varepsilon^{\prime}=\varepsilon\left\{\|Q\|(2+\varepsilon)+(1+\varepsilon)^{2}\right\} .
\end{gathered}
$$

By (8.45), (8.46) and (8.42), we have

$e^{-2 \varrho N}\left(\varepsilon+22 b(\|\Phi\|) \varepsilon^{\prime}\right)>\left\|L^{N}\left\{\left(K_{N}^{*}\right)^{-1} K_{(N, N)}^{*} Q^{(2 N)} K_{(N, N)} K_{N}^{-1}\right\}\right\|$.

By (6.5) and $K_{(N, N)} \in \mathfrak{A}([-2 N, 2 N])$, we have

$$
\begin{aligned}
L^{N}\left\{\left(K_{N}^{*}\right)^{-1} K_{(N, N)}^{*} Q^{(2 N)} K_{(N, N)} K_{N}^{-1}\right\} \tau_{c}(N) Q_{2} \\
=L^{N}\left\{\left(K_{N}^{*}\right)^{-1} K_{(N, N)}^{*} Q^{(2 N)} Q_{2} K_{(N, N)} K_{N}^{-1}\right\} .
\end{aligned}
$$

By (8.49), (8.50), (8.47) and (8.42), we have

$$
\left\|L^{N}\left(Q Q_{2}\right)\right\| e^{2 \varrho N}<\left\|Q_{2}\right\|\left\{\varepsilon+44 b(\|\Phi\|) \varepsilon^{\prime}\right\} .
$$

Hence, for $N>L\left([1+44 b(\|\Phi\|)\{4+3\|Q\|\}]^{-1} \varepsilon\right), \varepsilon<1$, we have

$$
\left|v\left(Q Q_{2}\right)\right|=\left|v\left(L^{N}\left(Q Q_{2}\right)\right)\right|<\varepsilon e^{-2 \varrho N}\left\|Q_{2}\right\|
$$

for any $Q_{2} \in \mathfrak{A}(Z \backslash[-2 N, 2 N])$.

For general $Q$, apply (8.52) for $Q-v(Q)$ and we obtain

$$
\left|v\left(Q Q_{2}\right)-v(Q) v\left(Q_{2}\right)\right|<\varepsilon e^{-2 \varrho N}\left\|Q_{2}\right\| .
$$

We now apply (8.53) for $F^{*} Q Q_{2} F$, and $F^{*} Q F$. Although one $F$ is on the right of $Q_{2}$, the same formula (8.53) holds for sufficiently large $N$ because $\left\|\left[Q_{2}, F\right]\right\|$ has a similar bound due to Lemma 5.2. Therefore

$$
\begin{gathered}
\left|v\left(F^{*} Q Q_{2} F\right)-v\left(F^{*} Q F\right) v\left(Q_{2}\right)\right|<\varepsilon e^{-2 \varrho N}\left\|Q_{2}\right\|, \\
\left|v\left(F^{*} Q_{2} F\right)-v\left(F^{*} F\right) v\left(Q_{2}\right)\right|<\varepsilon e^{-2 \varrho N}\|Q\| .
\end{gathered}
$$

By (8.27), we have

$$
e^{2 \varrho N}\left|\varphi_{\Phi}\left(Q Q_{2}\right)-\varphi_{\Phi}(Q) \varphi_{\Phi}\left(Q_{2}\right)\right|<\varepsilon v\left(F^{*} F\right)^{-1}(1+\|Q\|)\left\|Q_{2}\right\| .
$$

This proves the uniform exponential clustering property of $\varphi_{\Phi}$. Q.E.D. 


\section{§ 9. Analyticity}

Lemma 9.1. If $\Phi \in \mathfrak{A}([0, r])$ and $Q \in \mathfrak{A}([0, N])$ are holomorphic function of $\zeta=\left(\zeta_{1} \ldots \zeta_{n}\right)$ in a domain $D$ where $r$ and $N$ are fixed, then $E_{r}(Q ; H(I))$ is holomorphic in $\zeta$ in $D$ with respect to \|\|$_{1, x}, x>1$ and \|\| .

Proof. Let $\zeta^{(0)} \in D$ and $\Phi=\Sigma \Phi_{m}\left(\zeta-\zeta^{(0)}\right)^{m}, Q=\Sigma Q_{m}\left(\zeta-\zeta^{(0)}\right)^{m}$, $m=\left(m_{1} \ldots m_{n}\right), \quad\left(\zeta-\zeta^{(0)}\right)^{m}=\prod_{j}\left(\zeta_{j}-\zeta_{j}^{(0)}\right)^{m_{j}}$. Then $\Sigma\left\|\Phi_{m}\right\|\left|\zeta-\zeta^{(0)}\right|^{m} \leqq a_{\Phi}$, $\Sigma\left\|Q_{m}\right\|\left|\zeta-\zeta^{(0)}\right|^{m} \leqq a_{Q}$ uniformly in $\zeta$ in a neighbourhood of $\zeta^{(0)}$.

We substitute these expansions into estimates in Theorem 4.2 and Lemma 5.2. The estimate there only uses the property $Q \in \mathfrak{A}([0, r])$ and their norms. Hence all estimates holds when $\|\Phi\|$ and $\|Q\|$ are replaced by $a_{\Phi}$ and $a_{Q}$. In particular, $E_{r}(Q ; H(I))=\Sigma E_{m}\left(\zeta-\zeta^{(0)}\right)^{m}$ and

$$
\begin{gathered}
\sum_{m}\left\|E_{m}\right\|\left|\zeta-\zeta^{(0)}\right|^{m} \leqq \exp \left\{F_{N}\left(2 a_{\Phi}\right) a_{Q}\right\}, \\
\sum_{m}\left\|E_{m}\right\|_{N+L}\left|\zeta-\zeta^{(0)}\right|^{m} \leqq C_{N}^{\prime} \delta_{L}\left(a_{\Phi}\right),
\end{gathered}
$$

where $C_{N}^{\prime}$ now depends on $a_{\Phi}, a_{Q}$ and $N$. Therefore $E_{r}(Q ; H(I))$ has a convergent power series expansion at $\zeta^{(0)}$ and is holomorphic in $\zeta$.

Lemma 9.2. Let $\mathfrak{B}$ be a Banach space with a norm $\|Q\|$ for $Q \in \mathfrak{B}$. Let $\mathscr{L}(\zeta)$ be a bounded linear operator on $\mathfrak{B}$, holomorphic in $\zeta=\left(\zeta_{1} \ldots \zeta_{n}\right)$ in a neighbourhood $D$ of a real point $\xi_{0}, \mathscr{L}(\xi) h(\xi)=\lambda(\xi) h(\xi)$ for real $\xi$ in $D, h(\xi) \in \mathfrak{B}, \lambda(\xi)>0$. Let $v_{\xi}$ be in the dual $\mathfrak{B}^{*}$ of $\mathfrak{B}, E_{\xi} Q=v_{\xi}(Q) h(\xi)$, $v_{\xi}(h(\xi))=1, v_{\xi}(\mathscr{L}(\xi) Q)=\lambda(\xi) v_{\xi}(Q)$ for real $\xi$ in $D$ and $Q \in \mathfrak{B}$. Assume that there exists $\mu_{\xi}<\lambda(\xi)$ satisfying

$$
\lim _{N \rightarrow \infty} \mu_{\xi}^{-N}\left\|\mathscr{L}(\xi)^{N}\left(1-E_{\xi}\right)\right\|=0 .
$$

Let $Q_{0} \in \mathfrak{B}$ be fixed and $v_{\xi}\left(Q_{0}\right)=1$. Then there exist extensions $h(\zeta), \lambda(\zeta)$ and $v_{\zeta}$ for $\zeta$ in some neighbourhood $D^{\prime}$ of $\xi_{0}$, such that $\lambda(\zeta)$ is a holomorphic function of $\zeta, h(\zeta)$ is a $\mathfrak{B}$ valued holomorphic function of $\zeta$ and $v_{\zeta}$ is a $\mathfrak{B}^{*}$ valued holomorphic function of $\zeta$.

Proof. The series

$$
(Z-\mathscr{L}(\xi))^{-1}=(Z-\lambda(\xi))^{-1} E_{\xi}+\sum_{n=1}^{\infty} Z^{-n} \mathscr{L}(\xi)^{n-1}\left(1-E_{\xi}\right)
$$

is convergent for $|Z|>\mu_{\xi}, Z \neq \lambda(\xi)$, by (9.3) and is the inverse of $Z-\mathscr{L}(\xi)$. Let $\mu_{1}>\mu_{\xi_{0}}, \lambda\left(\xi_{0}\right)-\mu_{1}>0$. If $|Z| \geqq \mu_{1}$, we have

$$
\begin{aligned}
\left\|\left(Z-\mathscr{L}\left(\xi_{0}\right)\right)^{-1} \mid\right\| \leqq & \left|Z-\lambda\left(\xi_{0}\right)\right|^{-1}\left\|E_{\xi_{0}}\right\| \mid \\
& +\sum_{n=1}^{\infty} \mu_{1}^{-n}\left\|\mathscr{L}\left(\xi_{0}\right)^{n-1}\left(1-E_{\xi_{0}}\right)\right\| \| .
\end{aligned}
$$


Let $\delta=\sum_{n=1}^{\infty} \mu_{1}^{-n}\left\|\mathscr{L}\left(\xi_{0}\right)^{n-1}\left(1-E_{\xi_{0}}\right)\right\| \|$. By Lemma 3.7, we have

$\left(Z-\mathscr{L}\left(\xi_{0}\right)-\Delta\right)^{-1}=\sum_{n=1}^{\infty}\left\{\left(Z-\mathscr{L}\left(\xi_{0}\right)\right)^{-1} \Delta\right\}^{n-1}\left(Z-\mathscr{L}\left(\xi_{0}\right)\right)^{-1}$

provided that

$$
\|\Delta\|<\left(\delta+\delta^{\prime}\right)^{-1},\left|Z-\lambda\left(\xi_{0}\right)\right|>\left\|\left|E_{\xi_{0}}\right|\right\|\left(\delta^{\prime}\right)^{-1} .
$$

If $\Delta$ is a holomorphic function of $\zeta,(9.6)$ is holomorphic in $\zeta$ if $\|\Delta\|$ $<\left(\delta+\delta^{\prime}\right)^{-1}$. (The uniform limit of a holomorphic function is holomorphic.) Let $S\left(\delta^{\prime}\right)$ be the circle of radius $2\left\|E_{\xi_{0}}\right\|\left(\delta^{\prime}\right)^{-1}$ with the center $\lambda\left(\xi_{0}\right)$. Let $\Delta=\mathscr{L}(\zeta)-\mathscr{L}\left(\xi_{0}\right)$. Define

$$
E_{\zeta}^{\prime}=(2 \pi i)^{-1} \oint_{S\left(\delta^{\prime}\right)}\left(Z-\mathscr{L}\left(\xi_{0}\right)-\Delta\right)^{-1} \mathrm{dZ}
$$

Provided that

$$
2\|\| E_{\xi_{0}}\left\|\left(\delta^{\prime}\right)^{-1} \leqq \lambda\left(\xi_{0}\right)-\mu_{1},\right\| \Delta \| \leqq\left(\delta+\delta^{\prime}\right)^{-1},
$$

we have from (9.4) and (9.8)

$$
E_{\zeta}^{\prime}=E_{\xi_{0}} \quad \text { if } \quad \zeta=\zeta_{0} .
$$

Since $\left(Z-\mathscr{L}\left(\xi_{0}\right)-\Delta\right)^{-1}$ is holomorphic in $\zeta$ as long as $Z \in S\left(\delta^{\prime}\right)$ and (9.9) holds, $E_{\zeta}^{\prime}$ is holomorphic in $\zeta$.

As is easily seen the dimension of $E_{\zeta}^{\prime} \mathfrak{B}$ is continuous in $\zeta:$ If $\operatorname{dim} E_{a}^{\prime} \mathfrak{B}$ $<\operatorname{dim} E_{b}^{\prime} \mathfrak{B}, \operatorname{dim} E_{a}^{\prime} \mathfrak{B}<\infty$ then by an orthogonalization procedure there exists $\psi \in E_{b}^{\prime} \mathfrak{B}, \psi \neq 0$ such that $E_{a}^{\prime} \psi=0$, which contradict the continuity. Therefore $\operatorname{dim} E_{\zeta}^{\prime} \mathfrak{B}=\operatorname{dim} E_{\xi_{0}} \mathfrak{B}=1$ as long as $E_{\zeta}^{\prime}$ is holomorphic.

This then implies $\mathscr{L}(\zeta) E_{\zeta}^{\prime}=\lambda^{\prime}(\zeta) \mathrm{E}_{\zeta}$ because $\mathscr{L}(\zeta)$ commutes with $E_{\zeta}^{\prime}$. Since $\lambda^{\prime}(\zeta)=v_{\xi_{0}}\left(\mathscr{L}(\zeta) E_{\zeta}^{\prime} h\left(\xi_{0}\right)\right) v_{\xi_{0}}\left(E_{\zeta}^{\prime} h\left(\xi_{0}\right)\right)^{-1}, \lambda^{\prime}(\zeta)$ is holomorphic in $\zeta$ as long as $E_{\zeta}^{\prime}$ is holomorphic and $v_{\xi 0}\left(E_{\zeta}^{\prime} h\left(\xi_{0}\right)\right) \neq 0$. The latter is guaranteed in a neighbourhood of $\zeta=\xi_{0}$ because $v_{\xi_{0}}\left(E_{\xi_{0}} h\left(\xi_{0}\right)\right)=1$. Let $h^{\prime}(\zeta)=E_{\zeta}^{\prime} Q_{0}$. It is holomorphic and $h^{\prime}(\zeta)=h\left(\xi_{0}\right)$ when $\zeta=\xi_{0}$. Finally, let $v_{\zeta}^{\prime}(Q)$ $=v_{\xi_{0}}\left(E_{\zeta}^{\prime} Q\right) v_{\xi_{0}}\left(h^{\prime}(\zeta)\right)^{-1}$. It is holomorphic as long as $v_{\xi_{0}}\left(h^{\prime}(\zeta)\right) \neq 0$, and $v_{\zeta}^{\prime}(Q)=v_{\xi_{0}}(Q)$ when $\zeta=\xi_{0}$. Since $v_{\xi_{0}}\left(h\left(\xi_{0}\right)\right)=1, v_{\xi_{0}}\left(h^{\prime}(\zeta)\right) \neq 0$ in some neighbourhood of $\zeta=\xi_{0}$.

By $(9.4), \lambda(\xi)$ is the only singularity of $(Z-\mathscr{L}(\xi))^{-1}$ outside of a circle of radius $\mu_{\xi}$. If $\zeta$ is in sufficiently small neighbourhood of $\xi_{0}$ so that $\|\Delta\|$ is small, then $\lambda^{\prime}(\zeta)$ is the only singularity of $(Z-\mathscr{L}(\zeta))^{-1}$ outside of a circle of radius $\mu_{1}$. Hence $\lambda^{\prime}(\xi)=\lambda(\xi)$ for real $\xi$ in a neighbourhood of $\xi_{0}$. The expansion (9.4) then proves $E_{\xi}^{\prime}=E_{\xi}, h^{\prime}(\xi)=h(\xi)$ and $v_{\xi}^{\prime}=v_{\xi}$. Q.E.D.

$\mathscr{L}(\zeta) E_{\zeta}^{\prime}=\lambda^{\prime}(\zeta) E_{\zeta}^{\prime}$ implies $\mathscr{L}(\zeta) h^{\prime}(\zeta)=\lambda^{\prime}(\zeta) h^{\prime}(\zeta)$ and $\mathscr{L}(\zeta)^{*} v_{\zeta}^{\prime}=\lambda^{\prime}(\zeta) v_{\zeta}^{\prime}$

11 Commun. math Phys., Vol 14 
Lemma 9.3. $\varphi_{\Phi}(Q), Q \in \mathfrak{U}_{M, x}, x>1$ and $P(\Phi)$ is holomorphic in $\Phi$.

Proof. Let $\Phi$ be holomorphic in $\zeta$ and hermitian when $\zeta$ is real. Let

$$
\mathscr{L}(\zeta) Q=\tau_{c}(1) d^{-2} \operatorname{tr}_{[0,1]}\left(K(\zeta)^{*} Q K(\zeta)\right)
$$

where $K(\zeta)$ is defined by (6.2) (6.4) where $\Phi$ is now a holomorphic function of $\zeta . K(\zeta) \in \mathfrak{A}_{M, x}$ for any $x>1$ and is holomorphic with respect to \|\|$_{M, x}$ by Lemma 9.1. Since $\mathfrak{A}_{M, x}$ is a $*$-Banach algebra, this implies that $K(\zeta)^{*} Q K(\zeta)$ is holomorphic in $\zeta$ with respect to \|\|$_{M, x}$. Since $\left\|\tau_{c}(1) \mathrm{d}^{-2} \operatorname{tr}_{[0,1]} Q\right\|_{1, x} \leqq\|Q\|_{1, x}, \mathscr{L}(\zeta)$ is also holomorphic in $\zeta$.

Now Lemma 9.2 is applicable for $\mathfrak{B}=\mathfrak{A}_{M, x}$ and $\mathscr{L}(\zeta)$. We see that $\lambda, v$ and $h$ are holomorphic in $\zeta . F \in \mathfrak{U}_{M, x}$ is also holomorphic in $\zeta$. Hence $P(\Phi)$ and $\varphi_{\Phi}$ are also holomorphic in $\zeta$. Q.E.D.

Remark 9.4. The present proof of analyticity is applicable to the one dimensional classical spin lattice with an exponentially decreasing potentials. For higher dimensional quantum lattice, the analyticity for low activity is proved in $[6,7,8]$.

\section{§ 10. Factor States and Extremal KMS States}

Lemma 10.1. Let $\mathfrak{A}$ be a $C^{*}$-algebra, $\mathfrak{H}_{n} \subset \mathfrak{A}, n \in Z, \mathfrak{A}(I)$ be the $C^{*}$-algebra generated by $\mathfrak{A}_{n}, n \in I, \mathfrak{H}(Z)=\mathfrak{A}, Q \in \mathfrak{A}_{n}$ commutes with $Q^{\prime} \in \mathfrak{U}_{n^{\prime}}$ for $n \neq n^{\prime}$ and $\pi$ be a representation of $\mathfrak{2}$ such that $\pi(\mathfrak{U}(I))$ is a factor of type I for any finite I. Then $\pi(\mathfrak{U}(I))^{\prime} \cap \pi(\mathfrak{Q})^{\prime \prime}=\pi(\mathfrak{U}(Z \backslash I))^{\prime \prime}$ for any finite $I$.

Proof. Let $\mathfrak{A}_{0}(I)$ be the *-algebra generated by $\mathfrak{A}_{n}, n \in I$. Let $u_{i j}$ be the matrix unit of $\pi[\mathfrak{U}(I)]$. Then $Q \in \pi(\mathfrak{H})^{\prime \prime}$ is written as $Q=\Sigma u_{i j} Q_{i j}$, $Q_{i j}=\sum_{k} u_{k i} Q u_{j k} \in \pi(\mathfrak{2}(I))^{\prime}$. Since $\pi(\mathfrak{U})^{\prime \prime}$ is the $\sigma$-weak closure of $\pi\left(\mathfrak{H}_{0}(Z)\right)$ and $Q_{i}^{\prime j} \in \pi\left(\mathfrak{H}_{0}(Z \backslash I)\right)$ for $Q^{\prime} \in \pi\left(\mathfrak{H}_{0}(Z)\right)$, we have $Q_{i j} \in \mathfrak{H}(Z \backslash I)$. If $Q \in \pi(\mathfrak{H}(I))^{\prime}$, then $Q_{i j}=\delta_{i j} Q$. Q.E.D.

This is essentially Lemma 2.3 of [10]. This lemma for somewhat more general case follows from Lemma 3.2 of [4].

A state $\varphi$ of $A$ in Lemma 10.1 is said to be uniformly clustering if there exists finite $I$ for given $\varepsilon>0$ and $Q \in \mathfrak{Q}$ such that

$$
\left|\varphi\left(Q Q_{1}\right)-\varphi(Q) \varphi\left(Q_{1}\right)\right|<\varepsilon\left\|Q_{1}\right\|
$$

for every $Q_{1} \in \mathfrak{A}(Z \backslash I)$. This condition may be replaced by a number of equivalent conditions. We may require (2.1) for any given $Q \in \mathfrak{A}_{0}(Z)$ and for any $Q_{1} \in \mathfrak{A}_{0}(Z \backslash I)$. If we denote the representation of $\mathfrak{A}$ associated with the state $\varphi$ by $\pi_{\varphi}$, then another equivalent condition is

$$
\left|\left(\Psi_{1}, \pi_{\varphi}\left(Q_{1}\right) \Psi_{2}\right)-\left(\Psi_{1}, \Psi_{2}\right) \varphi\left(Q_{1}\right)\right|<\varepsilon\left\|Q_{1}\right\|
$$


for any given $\Psi_{1}, \Psi_{2}$. In fact (2.1) is a special case of (2.2) with $\Psi_{1}=\pi_{\varphi}\left(Q^{*}\right) \Omega_{\varphi}, \Psi_{2}=\Omega_{\varphi}$. On the other hand, (2.2) for a dense set of vectors $\Psi_{1}=\pi_{\varphi}\left(Q_{a}^{*}\right) \Omega_{\varphi}, \pi_{2}=\pi_{\varphi}\left(Q_{b}\right) \Omega_{\varphi}, Q_{a}, Q_{b} \in \mathfrak{A}_{0}(Z)$ follows from (2.1) for sufficiently big $I$ such that $Q_{a}, Q_{b} \in \mathfrak{A}(I)$. Hence (2.2) holds for every given $\Psi_{1}$ and $\Psi_{2}$ for sufficiently large $N$. The condition (2.2) is equivalent to (2.2) with the specialization $\Psi_{1}=\Psi_{2}$.

Lemma 10.2. Let $\mathfrak{A}$ be as in Lemma 10.1 where $\pi=\pi_{\varphi}$ is canonically associated with a given state $\varphi$. Then $\pi_{\varphi}(A)^{\prime \prime}$ is a factor if and only if $\varphi$ is uniformly clustering.

Proof. The only if part is in Lemma 4.12 of [1]. For the if part, any central element $S$ of $\pi_{\varphi}(\mathfrak{U})^{\prime \prime}$ is in $\pi_{\varphi}(\mathfrak{U}(Z \backslash I))^{\prime \prime}$ for any $I$. Given $\varepsilon$, we choose $I$ satisfying (2.2) and then $Q_{1} \in \mathfrak{U}(Z \backslash I)$ such that

$$
\left|\left(\psi_{1},\left(\pi_{\varphi}\left(Q_{1}\right)-S\right) \Psi_{2}\right)\right|<\varepsilon, \quad\left|\left(\Psi_{1}, \Psi_{2}\right)\left\{\varphi\left(Q_{1}\right)-\left(\Omega_{\varphi}, S \Omega_{\varphi}\right)\right\}\right|<\varepsilon .
$$

Then we have $\left|\left(\Psi_{1}, S \Psi_{2}\right)-\left(\Psi_{1}, \Psi_{2}\right)\left(\Omega_{\varphi}, S \Omega_{\varphi}\right)\right|<3 \varepsilon$. Since $\varepsilon$ is arbitrary, $S=\left(\Omega_{\varphi}, S \Omega_{\varphi}\right)$ 1. Q.E.D.

This is essentially Theorem 2.5 of [10]. It is used under slightly more general circumstances around Eq. (3.6) of [4]. Lemma 10.2 and Lemma 10.1 are also derived in [9] in connection with a characterization of pure phase in both classical and quantum statistical mechanics.

The central decomposition of states into factor states always exists and is unique [14]. If the state is a KMS state, then the factor states are KMS states at least if $A$ is separable (Corollary 3.7 [2]). Further Theorem 4.1 in [2] essentially implies, though not explicitly stated, the following theorem.

Theorem 10.3. A KMS state is a factor state if and only if it is an extremal KMS state.

Proof. Let the representation $\pi_{\varphi}$ of a $C^{*}$-algebra $\mathfrak{A}$ and a cyclic vector $\Omega_{\varphi}$ in the representation space $\mathfrak{G}_{\varphi}$ of $\pi_{\varphi}$ be canonically associated with a KMS state $\varphi\left(\right.$ i.e. $\left.\varphi(Q)=\left(\Omega_{\varphi}, \pi_{\varphi}(Q) \Omega_{\varphi}\right)\right)$. It is shown in [3] that the center of $\pi_{\varphi}(\mathfrak{Q})^{\prime \prime}$ is elementwise invariant under time translation. For any central projection $F \neq 0, \varphi_{F}(Q)=\left(F \Omega_{\varphi}, \pi_{\varphi}(Q) F \Omega_{\varphi}\right)\left\|F \Omega_{\varphi}\right\|^{-2}$ is a KMS state $\left(\left\|F \Omega_{\varphi}\right\| \neq 0\right.$ always) and is different from $\varphi(Q)$ unless $F=1$. Hence if $\varphi$ is not a factor state, $\varphi=\lambda \varphi_{F}+(1-\lambda) \varphi_{1-F}, 0<\lambda=\left\|F \Omega_{\varphi}\right\|^{2}<1$ and $\varphi$ is not an extremal KMS state.

Conversely, let $\varphi=\lambda \varphi_{1}+(1-\lambda) \varphi_{2}, 0<\lambda<1$ where $\varphi_{1}$ and $\varphi_{2}$ are 'KMS states. There exists an operator $F \geqq 0$ in $\pi_{\varphi}(\mathfrak{H})^{\prime}$ such that $\lambda \varphi_{1}(Q)$ $=\left(\Omega_{\varphi}, \pi_{\varphi}(Q) F \Omega_{\varphi}\right)$. Since $\varphi_{1}\left(\tau_{T}(t) Q\right)=\varphi_{1}(Q)$ by assumption, $\left(\pi_{\varphi}\left(Q_{1}\right) \Omega_{\varphi}\right.$, $\left.F \pi_{\varphi}\left(Q_{2}\right) \Omega_{\varphi}\right)=\left(\pi_{\varphi}\left(\tau_{T}(t) Q_{1}\right) \Omega_{\varphi}, F \pi_{\varphi}\left(\tau_{T}(t) Q_{2}\right) \Omega_{\varphi}\right)$, from which we have $F=U_{\varphi}(t)^{*} F U_{\varphi}(t)$. Namely $F \in R_{1}^{\prime}$ in the notation of [1]. By the $K M S$ 
condition on $\varphi_{1}$, we have $\left(\Omega_{\varphi}, \hat{Q}_{1} \hat{Q}_{2}\left(f_{0}\right) F \Omega_{\varphi}\right)=\left(\Omega_{\varphi}, \hat{Q}_{2}\left(f_{1}\right) \hat{Q}_{1} F \Omega_{\varphi}\right)$. This is assumed for $\hat{Q}_{1} \in \pi_{\varphi}(\mathfrak{U})$ and hence it holds for $\hat{Q}_{1} \in \pi_{\varphi}(\mathfrak{A})^{\prime \prime}$ by the weak closure. Rewriting the equation as $\left(\Omega_{\varphi}, \hat{Q}_{1}\left(f_{1}^{\prime}\right) \hat{Q}_{2} F \Omega_{\varphi}\right)$ $=\left(\Omega_{\varphi}, \hat{Q}_{2} \hat{Q}_{1}\left(f_{0}^{\prime}\right) \Omega_{\varphi}\right)$ with $f_{0}^{\prime}(t)=f_{1}(-t)$, we see that it also holds for $\hat{Q}_{2} \in \pi_{\varphi}(\mathfrak{U})^{\prime \prime}$, if it is assumed for $\hat{Q}_{2} \in \pi_{\varphi}(\mathfrak{A})$. Now we restrict our attention to $E_{0} \mathfrak{H}_{\varphi}$ of $t$ invariant vectors in $\mathfrak{H}_{\varphi} . \hat{\varphi}$ is a cyclic and separating trace of $E_{0} \pi_{\varphi}(\mathfrak{A})^{\prime \prime} E_{0}$, where $\hat{\varphi}(Q)=\left(\Omega_{\varphi}, Q \Omega_{\varphi}\right)$ for $Q \in \mathscr{B}\left(\mathfrak{H}_{\varphi}\right)$. This property should also holds for $\hat{\varphi}$ similarly defined from $\varphi_{1}$. Namely $\hat{\varphi}_{1}$ is a cyclic and separating trace of $F E_{0} \pi_{\varphi}(\mathfrak{Q})^{\prime \prime} F E_{0}$ in $F E_{0} \mathfrak{H}_{\varphi}$. This implies that $F E_{0}$ must be in the center of $E_{0} \pi_{\varphi}(\mathfrak{Q})^{\prime \prime} E_{0}$ by an easy calculation. The argument in the proof of Theorem 4.1 of [2] then shows that there must be a central element $F_{1}$ of $\pi_{\varphi}(\mathfrak{U})^{\prime \prime}$ such that $F E_{0}=F_{1} E_{0}$. Since $R_{1}^{\prime} \rightarrow R_{1}^{\prime} E_{0}$ is an isomorphism, $F=F_{1}$ and $F$ is a non trivial central element of $\pi_{\varphi}(\mathfrak{U})^{\prime \prime}$. Namely $\pi_{\varphi}(\mathfrak{U})^{\prime \prime}$ is not a factor if $\varphi$ is not an extremal KMS state. Q.E.D.

The decomposition of a KMS states into extremal KMS states coincides with the decomposition into extremal time translation invariant states if and only if $\pi_{\varphi}$ is $\eta$ abelian where $\eta$ is taken as the mean $(2 T)^{-1} \int_{-T}^{T} d T$ as $T \rightarrow \infty$. ([2].)

For the one dimensional quantum spin lattice, Lemma 10.2 and Theorem 10.3 are applicable and $\varphi_{\Phi}$ is a factor state and is an extremal $K M S$ state because it is uniformly clustering. The asymptotic abelaian property relative to the lattice translation also implies that the factor state $\varphi_{\Phi}$ is an extremal lattice translation invariant state.

Acknowledgement. The author thanks Director L. Motchane and the members of the Institut des Hautes Etudes Scientifiques for their kind hospitality and Dr. Ruelle for discussions.

\section{References}

1. Araki, H., and E. J. Woods: A complete Boolean lattice of type I factors. Publ. Res. Inst. Math. Sci. Kyoto Univ. Ser. A, 2, 157-242 (1966).

2. - , and H. Miyata: On KMS boundary condition. Publ. Res. Inst. Math. Sci. Kyoto Univ. Ser. A, 4, 373-385 (1968).

3. - Multiple time analyticity of a quantum statistical states satisfying the KMS boundary condition. Publ. Res. Inst. Math. Sci. Kyoto Univ. Ser. A, 4, 361-371 (1968).

4. - A classification of factors II. Publ. Res. Math. Sci. Kyoto Univ. Ser. A, 4, 585-593 (1968).

5. Fujiwara, I.: Operator calculus of quantized operator. Progr. Theoret. Phys. 7, $433-448$ (1952).

6. Galavotti, G., S. Miracle-Sole, and D. W. Robinson: Analyticity properties of the anisotropic Heisenberg model. Commun. Math. Phys. 10, 311-324 (1968).

7. Ginibre, J.: Reduced density matrices of the anisotropic Heisenberg model. Commun. Math. Phys. 10, 140-154 (1968). 
8. Greenberg, W.: Correlation functionals of infinite volume quantum spin systems. Commun. Math. Phys. 11, 314-320 (1969).

9. Landford III, O. E., and D. Ruelle: Observables at infinity and states with short range correlation in statistical mechanics. (preprint).

10. Powers, R. T.: Representations of uniformly hyperfinite algebras and their associated von Neumann rings. Ann. of Math. 86, 138-171 (1967).

11. Robinson, D. W.: Statistical mechanics of quantum spin system II. Commun. Math. Phys. 7, 337-348 (1968).

12. Ruelle, D.: Statistical mechanics of a one dimensional lattice gas. Commun. Math. Phys. 9, 267-278 (1968).

13. Streater, R. F.: The Heisenberg ferromagnet as a quantum field theory. Commun. Math. Phys. 6, 233-247 (1967).

14. Wils, W.: Désintégration centrale des formes positives des $C^{*}$-algebres. (preprint).

H. Araki

Research Institute for Mathematical Sciences

Kyoto University

Kyoto, Japan 